\title{
WINNING THE NIST CONTEST: A SCALABLE AND GENERAL APPROACH TO DIFFERENTIALLY PRIVATE SYNTHETIC DATA
}

\author{
RYAN MCKENNA, GEROME MIKLAU, AND DANIEL SHELDON
}

College of Information \& Computer Sciences, The University of Massachusets, Amherst, MA 10002

e-mail address: rmckenna@cs.umass.edu

College of Information \& Computer Sciences, The University of Massachusets, Amherst, MA 10002

e-mail address: miklau@cs.umass.edu

College of Information \& Computer Sciences, The University of Massachusets, Amherst, MA 10002

e-mail address: sheldon@cs.umass.edu

\begin{abstract}
We propose a general approach for differentially private synthetic data generation, that consists of three steps: (1) select a collection of low-dimensional marginals, (2) measure those marginals with a noise addition mechanism, and (3) generate synthetic data that preserves the measured marginals well. Central to this approach is Private-PGM [42], a post-processing method that is used to estimate a high-dimensional data distribution from noisy measurements of its marginals. We present two mechanisms, NIST-MST and MST, that are instances of this general approach. NIST-MST was the winning mechanism in the 2018 NIST differential privacy synthetic data competition, and MST is a new mechanism that can work in more general settings, while still performing comparably to NIST-MST. We believe our general approach should be of broad interest, and can be adopted in future mechanisms for synthetic data generation.
\end{abstract}

\section{INTRODUCTION}

Data sharing within the modern enterprise is extremely constrained by privacy concerns. Privacy-preserving synthetic data is an appealing solution: it allows existing analytics workflows and machine learning methods to be used while the original data remains protected. But recent research has shown that unless a formal privacy standard is adopted, synthetic data can violate privacy in subtle ways $[17,24]$. Differential privacy offers such a formalism, and the problem of differentially private synthetic data generation has therefore received considerable research attention in recent years $[2,5,8,12,13,25,30,31,38,39,53-56,60,61,67,69,71,72]$.

In 2018, the National Institute of Standards and Technology (NIST) highlighted the importance of this problem by organizing the Differential Privacy Synthetic Data Competition [57]. This competition was the first of its kind for the privacy research community, and it encouraged privacy researchers and practitioners to develop novel practical mechanisms for

$$
\begin{array}{ll}
P_{\text {rivacy }} & \text { www.journalprivacyconfidentiality.org } \\
\text { DOl:10.29012/jpc.778 }
\end{array}
$$

(c) Ryan McKenna, Gerome Miklau, and Daniel Sheldon (cc) EY-No-ND Creative Commons (CC BY-NC-ND 4.0)

Key words and phrases: differential privacy, synthetic data, graphical models. Code to accompany this article is available at [41]. 


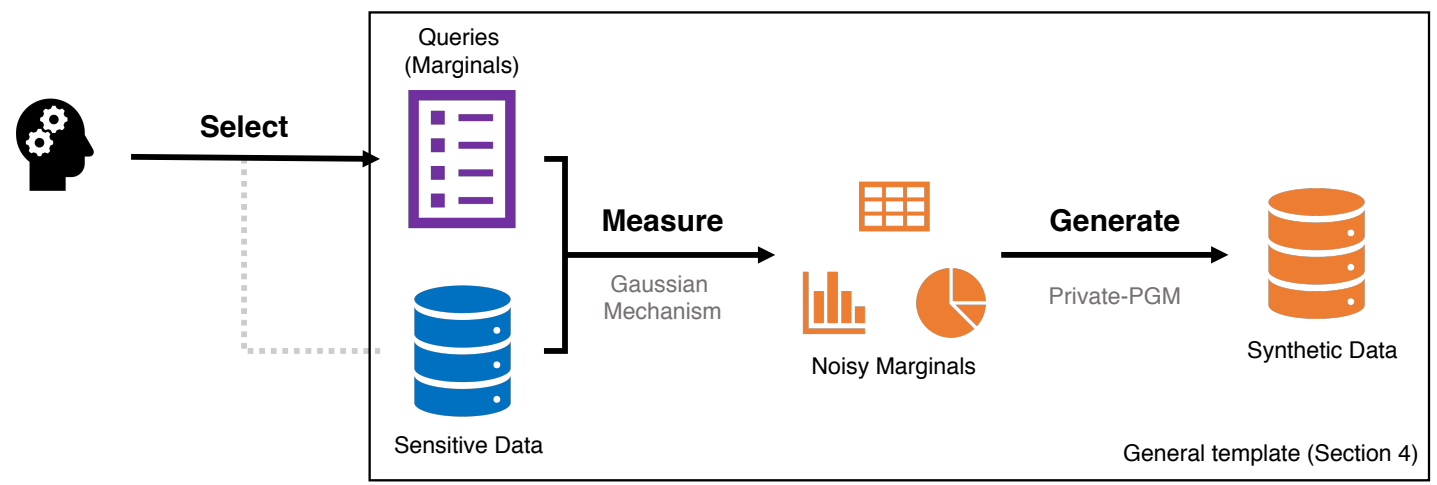

Figure 1. A general template for differentially private synthetic data generation. First, a collection of marginal queries is selected, either manually (e.g., by a domain expert) or automatically by an algorithm. Second, the Gaussian mechanism is used to measure those marginals while preserving differential privacy. Finally, Private-PGM is used to post-process the noisy marginals and generate a synthetic dataset that respects them.

this task. The competition consisted of three rounds of increasing complexity. In this paper we describe NIST-MST, the winning entry in the third and final round of the competition. Our algorithm is an instance of a general template for differentially private synthetic data generation that we believe will simplify design of future mechanisms for synthetic data.

Our approach to differentially private synthetic data generation consists of three highlevel steps, as show in Figure 1: (1) query selection, (2) query measurement and (3) synthetic data generation. For step (1), there are various ways to approach query selection; a domain expert familiar with the data and its use cases can specify the set of queries, or they can be automatically determined by an algorithm. The selected queries are important because they will ultimately determine the statistics for which the synthetic data preserves accuracy. For step (2), after the queries are fixed, they are measured privately with a noise-addition mechanism, in our case, with the Gaussian mechanism. In step (3), the noisy measurements are processed through Private-PGM [42], a post-processing method that can estimate a high-dimensional data distribution from noisy measurements and generate synthetic data.

This approach is similar in spirit to the widely studied select-measure-reconstruct paradigm for linear query answering under differential privacy $[3,15,16,28,34-37,37,40$, $46-48,58,59,62-66,68,70]$. However, the output is now synthetic data, rather than query answers. In addition, most existing methods from this paradigm suffer from the curse of dimensionality, and have trouble scaling to high-dimensional domains. Our approach is simple and modular but there are three main technical challenges to using it in practice. These are (1) identifying what statistics to measure about the dataset, (2) generating synthetic data that effectively preserves the measured statistics, and (3) overcoming the challenges of high-dimensional domains. Fortunately, Private-PGM solves problem (2) and (3) above, as long as the measured statistics only depend on the data through its low-dimensional marginals. This allows the mechanism designer to focus on problem (1), and frees them from the burden of figuring out how to generate synthetic data with differential privacy, allowing them instead to focus on what statistics to measure, based on what they want the synthetic data to preserve. Thus, we believe this approach to differentially private synthetic data, using Private-PGM, will be broadly applicable. 
In Figure 1, there is a dashed gray line connecting the select step with the sensitive data. This indicates that query selection may or may not depend on the sensitive data, but if it does, it must be via a differentially private mechanism. The rules of the NIST competition permitted the use of a public provisional dataset which the NIST-MST algorithm uses for query selection. The effectiveness of query selection relies on the similarity of the public data to the private data being synthesized. Since high quality provisional data may not always be available, we propose a variant of the algorithm, called MST, that does not require public data and instead uses a portion of the privacy budget to select measurements. The novelty of this algorithm is that it uses the data (privately) to select marginals to measure that support efficient synthesis in step (3). This extension leads to an algorithm that can be applied in a wider variety of settings. We show experimentally that, without the advantage of provisional data, it nevertheless performs comparably to NIST-MST.

This paper is organized as follows. In Section 2, we set up notation, state assumptions, and summarize relevant background in differential privacy. In Section 3, we summarize the important aspects of the competition. In Section 4, we describe the general template from Figure 1 in greater detail. In Section 5, we present NIST-MST, the winning mechanism from the competition, by building on the general template. In Section 6, we present MST, a novel mechanism inspired by NIST-MST that does not rely on the existence of public provisional data. We conclude with a simple experimental evaluation and discussion of results.

\section{BACKGROUND}

The algorithms described in this paper take as input a dataset, assumed to be a single table, and generate a synthetic dataset satisfying $(\epsilon, \delta)$-differential privacy. Below we provide the relevant background and notation on datasets, marginals, and differential privacy.

2.1. Data. The input is a dataset $D$ consisting of $m$ records, each containing potentially sensitive information about one individual. Each record has $d$ attributes $\mathcal{A}=\left\{A_{1}, \ldots, A_{d}\right\}$, and the domain of possible values for an attribute $A_{i}$ is denoted by $\Omega_{i}$. We assume $\Omega_{i}$ is finite and has size $\left|\Omega_{i}\right|=n_{i}$. The full domain of possible values is thus $\Omega=\Omega_{1} \times \cdots \times \Omega_{d}$ which has size $\prod_{i} n_{i}=n$. We use $\mathcal{D}$ to denote the set of all possible datasets, which is equal to $\mathcal{D}=\cup_{m=0}^{\infty} \Omega^{m}$.

2.2. Marginals. A marginal is a key statistic that captures low-dimensional structure in a high-dimensional data distribution. We will explain (in Section 3) that the evaluation metrics of the contest can be defined in terms of marginals computed on the dataset. In addition, our algorithms will privately measure selected marginals and use the resulting noisy measurements to construct synthetic data. More precisely, a marginal, for a set of attributes $C$, is a table that counts the number of occurrences of each combination of possible values for attributes $C$.

Definition 1 (Marginal). Let $C \subseteq \mathcal{A}$ be a subset of attributes, $\Omega_{C}=\prod_{i \in C} \Omega_{i}$, and $n_{C}=\left|\Omega_{C}\right|$. The marginal on $C$ is a vector $\mu \in \mathbb{R}^{n_{C}}$, indexed by domain elements $t \in \Omega_{C}$, such that each entry is a count, i.e., $\mu_{t}=\sum_{x \in D} \mathbb{1}\left[x_{C}=t\right]$. We let $M_{C}: \mathcal{D} \rightarrow \mathbb{R}^{n_{C}}$ denote the function that computes the marginal on $C$, i.e., $\mu=M_{C}(D)$. 
2.3. Differential privacy. Differential privacy $[19,20]$ protects individuals by bounding the impact any one individual can have on the output of an algorithm. This is formalized using the notion of neighboring datasets. Two datasets $D, D^{\prime} \in \mathcal{D}$ are neighbors (denoted $\left.D \sim D^{\prime}\right)$ if $D^{\prime}$ can be obtained from $D$ by adding or removing a single record.

Definition 2 (Differential Privacy [19]). A randomized mechanism $\mathcal{M}: \mathcal{D} \rightarrow \mathcal{R}$ satisfies $(\epsilon, \delta)$-differential privacy $(D P)$ if for any neighboring datasets $D \sim D^{\prime} \in \mathcal{D}$, and any subset of possible outputs $S \subseteq \mathcal{R}$,

$$
\operatorname{Pr}[\mathcal{M}(D) \in S] \leq \exp (\epsilon) \operatorname{Pr}\left[\mathcal{M}\left(D^{\prime}\right) \in S\right]+\delta .
$$

This definition requires that, on any two neighboring input databases, the difference in the output distributions of the randomized algorithm $\mathcal{M}$ is bounded by $e^{\epsilon}$, except with a small failure probability $\delta$. This failure probability $\delta$ is usually assumed to be cryptographically small; in the contest it was set to $\delta \approx 2 \cdot 10^{-12}$. The algorithms in this paper achieve differential privacy by repeated application of the Gaussian mechanism and the Exponential Mechanism, defined below:

Definition 3 (Gaussian Mechanism). Let $f: \mathcal{D} \rightarrow \mathbb{R}^{p}$ be a vector-valued function of the input data. The Gaussian Mechanism adds i.i.d. Gaussian noise with scale $\sigma$ to $f(D)$ :

$$
\mathcal{M}(D)=f(D)+\mathcal{N}\left(0, \sigma^{2} \mathbf{I}\right)
$$

Definition 4 (Exponential Mechanism). Let $q: \mathcal{D} \times \mathcal{R} \rightarrow \mathbb{R}$ be quality score function and $\epsilon$ be a parameter. Then the exponential mechanism outputs a candidate $r \in \mathcal{R}$ according to the following distribution:

$$
\operatorname{Pr}[\mathcal{M}(D)=r] \propto \exp (\epsilon \cdot q(D, r))
$$

To accurately analyze the privacy of multiple invocations of the Gaussian/Exponential mechanisms (i.e., to derive the $(\epsilon, \delta)$ parameters) we use the tools of Rényi Differential Privacy (RDP), a variant of differential privacy so named because it uses the Rényi divergence in the bound on a mechanism's output distributions for neighboring inputs.

Definition 5 (Rényi Differential Privacy [45]). A randomized mechanism $\mathcal{M}: \mathcal{D} \rightarrow \mathcal{R}$ satisfies $(\alpha, \gamma)$-Rényi differential privacy (RDP) for $\alpha \geq 1$ and $\gamma \geq 0$, if for any neighboring datasets $D \sim D^{\prime} \in \mathcal{D}$, we have:

$$
D_{\alpha}\left(\mathcal{M}(D) \| \mathcal{M}\left(D^{\prime}\right)\right) \leq \gamma
$$

where $D_{\alpha}(\cdot \| \cdot)$ is the Rényi divergence of order $\alpha$ between two probability distributions.

To analyze the privacy of the mechanisms above under Rényi-DP, we define the sensitivity of a vector-valued query as follows:

Definition 6 (Sensitivity). Let $f: \mathcal{D} \rightarrow \mathbb{R}^{p}$ be a vector-valued function of the input data. The $L_{2}$ sensitivity of $f$ is $\Delta_{f}=\max _{D \sim D^{\prime}}\left\|f(D)-f\left(D^{\prime}\right)\right\|_{2}$.

It is easy to verify that the $L_{2}$ sensitivity of any marginal function $M_{C}$ is 1 , regardless of the attributes in $C$. This is because one individual can only contribute a count of 1 to a single cell of the output vector. A single invocation of the Gaussian Mechanism satisfies Rényi-DP with parameters determined by the noise scale $\sigma$ and the sensitivity $\Delta_{f}$ of the function. Similarly, a single invocation of the Exponential Mechanism also satisfies Rényi-DP with parameters determined by $\epsilon$ and $\Delta_{q}$. 
Proposition 1 (Rényi-DP of the Gaussian Mechanism [21,45]). The Gaussian Mechanism applied to the function $f: \mathcal{D} \rightarrow \mathbb{R}^{p}$ satisfies $\left(\alpha, \alpha \frac{\Delta_{f}^{2}}{2 \sigma^{2}}\right)-R D P$ for all $\alpha \geq 1$.

Proposition 2 (Rényi-DP of the Exponential Mechanism [11,44]). The Exponential Mechanism applied to the quality score function $q: \mathcal{D} \times \mathcal{R} \rightarrow \mathbb{R}$ satisfies $(2 \epsilon \Delta, 0)-D P$ and $\left(\alpha, \alpha \frac{(2 \epsilon \Delta)^{2}}{8}\right)-R D P$ for all $\alpha \geq 1$, where $\Delta=\max _{r \in \mathcal{R}} \Delta_{q(\cdot, r)}$ is the maximum sensitivity of $q$.

Note that any mechanism that is $(\alpha, \alpha \rho)$-RDP for all $\alpha \geq 1$ is also $\rho$-zCDP [10] and viceversa. We rely on the following propositions to reason about multiple adaptive invocations of RDP mechanisms, and the translation between Rényi-DP and $(\epsilon, \delta)$-DP.

Proposition 3 (Adaptive Composition of RDP Mechanisms [45]). Let $\mathcal{M}_{1}: \mathcal{D} \rightarrow \mathcal{R}_{1}$ be $\left(\alpha, \gamma_{1}\right)-R D P$ and $\mathcal{M}_{2}: \mathcal{D} \times \mathcal{R}_{1} \rightarrow \mathcal{R}_{2}$ be $\left(\alpha, \gamma_{2}\right)-R D P$. Then the mechanism $\mathcal{M}=$ $\mathcal{M}_{2}\left(D, \mathcal{M}_{1}(D)\right)$ is $\left(\alpha, \gamma_{1}+\gamma_{2}\right)-R D P$.

Proposition 4 (RDP to DP [45]). If a mechanism $\mathcal{M}$ satisfies $(\alpha, \gamma)$-Rényi differential privacy, it also satisfies $\left(\gamma+\frac{\log (1 / \delta)}{\alpha-1}, \delta\right)$-differential privacy for all $\delta \in(0,1]$.

\section{Competition setup}

In this section we will summarize the format of the competition and the different components of the challenge problem. The competition consisted of three rounds of increasing complexity, but our focus is on the third round, which built on the previous two rounds.

3.1. Competition Format. Competitors were given approximately one month to design their differentially private synthetic data mechanism. The competition organizers provided contestants with a precise problem specification along with a "competitor pack", which included a provisional dataset to test and develop mechanisms, a file that contained domain information for each attribute in the dataset, a script to evaluate the quality of the synthetic data according to their custom scoring criteria, and a baseline mechanism. Each of these components will be described in detail in the subsequent sections. During the one-month competition period, competitors could submit the synthetic data produced by their mechanism to be scored and attain a spot on the provisional leaderboard. This was a good way to gauge how well other competitors were doing, although it was not an authoritative source as the submissions had not been vetted to ensure they satisfied differential privacy (e.g., someone could submit the true data and get a perfect score on the provisional leaderboard).

At the end of the competition period, competitors had to submit their source code along with a document describing the solution and a proof of privacy. A team of experts unknown to the competitors checked the final submitted algorithms to ensure that they satisfied $(\epsilon, \delta)$-differential privacy. This was done by checking the written description of the algorithm as well as the source code, to ensure there were no privacy violations or mistakes. After the mechanism was verified to be differentially private, its utility was evaluated on the final dataset (different from the provisional dataset) and performance results were added to a final leaderboard that would determine the ranking of solutions.

In the subsequent subsections, we will specify the details of each component of the problem - i.e., the dataset, the domain, and the evaluation criteria. 
3.2. Dataset. Algorithms were designed for and evaluated on data from the 1940 U.S. decennial census. The provisional dataset contained data for one state (Colorado) and the final holdout data was for a different state (unknown at the time of the competition). We remark that the provisional dataset was treated as public information. Therefore, any analysis and insights derived from it were not considered to violate privacy. However, solutions that used information from the provisional dataset too aggressively risked over-fitting, and scoring poorly on the final dataset. The provisional dataset contained 98 attributes and about 661 thousand records. All attributes were discrete, taking on values from the domain $\Omega_{i}=\left\{0, \ldots, n_{i}-1\right\}$. The value of $n_{i}$ for each attribute $i$ was provided in a separate specs file. The values of $n_{i}$ ranged from 2 (for binary attributes like SEX) to 10, 000, 000 (for numerical attributes like INCWAGE). The total number of possible database rows (i.e., the full domain size) was about $|\Omega|=5 \times 10^{205}$. We provide a full breakdown of the domain in Table 4 .

3.3. Evaluation metrics. The utility of the synthetic data was measured by how well it preserved key statistics in the ground truth data with respect to three main criteria, enumerated below. We state below the statistics that need to be preserved to score well, but not the exact formula for calculating the score. For the precise information, please refer to the official challenge problem statement [57]. Note that the scores for each evaluation metric were normalized to the same range, and averaged across the three metrics (with equal weights). Algorithms were evaluated at three privacy levels, with $\epsilon=0.3,1.0,8.0$ and $\delta \approx 2 \cdot 10^{-12}$, and these scores were averaged to obtain the final score. Computational efficiency was not taken into consideration; several of the solutions (including NIST-MST) required up to 10 minutes or more to run.

(1) 3-way Marginals. The synthetic data was evaluated by comparing its marginals with the marginals of the true data for 100 random triples of attributes, unknown to competitors at submission time. Therefore, a synthetic dataset $\tilde{D}$ scores well on this metric (in expectation) if $M_{C}(D) \approx M_{C}(\tilde{D})$ for all triples $C$. There are a total of $\left(\begin{array}{c}98 \\ 3\end{array}\right)=152096$ possible triples, so this evaluation criteria requires the synthetic data to preserve a large number of marginals to consistently score well.

(2) High-order conjunctions. The synthetic data was evaluated by looking at how well it preserved high-order conjunctions. Probabilistically, a high-order conjunction for a set of attributes $C \subseteq \mathcal{A}$ assumes the form $\operatorname{Pr}\left[\bigwedge_{i \in C}\left[t_{i} \in S_{i}\right] \mid t \sim D\right]$, where $S_{i} \subseteq \Omega_{i}$ is a subset of the domain for attribute $i$. This quantity can be expressed in terms of the marginal $\mu=M_{C}(D)$ via $\sum_{t \in S} \mu_{t}$, where $S$ is the Cartesian product of $S_{i}$ 's, i.e., $S=\prod_{i \in C} S_{i}$ and $t$ is a tuple restricted to the attributes in the set $C$. Synthetic data was evaluated on 300 random high-order conjunctions, where $C$ is generated with a simple random sample of the attributes $\mathcal{A}$ with selection probability 0.1 , and $S_{i}$ is a random subset of $\Omega_{i}$. There are a total of $2^{98} \approx 10^{29}$ possible choices for $C$, and the expected size of $C$ is $0.1 \cdot 98 \approx 10$. Even without accounting for the variability in $S_{i}$, it is clear that the number of statistics that need to be preserved is enormous.

(3) Income inequality and gender wage gap. The synthetic data was evaluated by how well it preserved statistics relating to income inequality and gender wage gap, broken down by city. While the precise details of this metric can be found in the official problem statement, to score well, it suffices for the synthetic data to be accurate with respect to the marginal on (SEX, CITY, INCWAGE). Unlike metrics (1) and (2), above, this metric is relatively easy to score well on, because it just requires preserving one marginal well. 


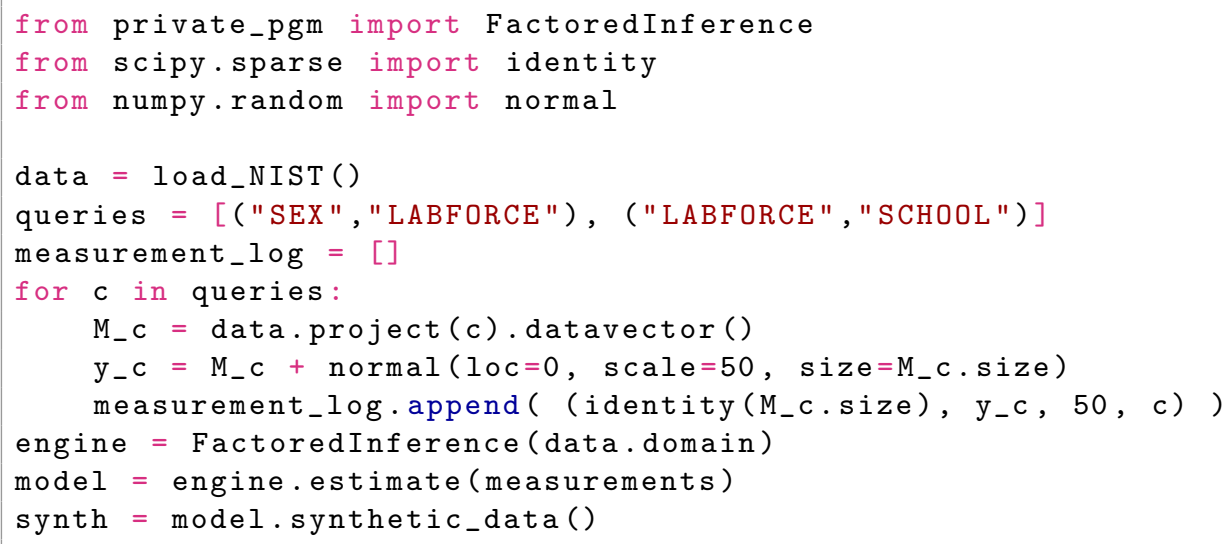

Figure 2. A demonstration of how to generate synthetic data with Private-PGM using real Python code. In this case, the selected marginals are (SEX, LABFORCE) and (LABFORCE, SCHOOL). In Lines 9-12, these marginals are measured with Gaussian noise to protect privacy. In Lines 14-16, Private-PGM takes these noisy measurements as input, estimates a model, and generates synthetic data. The Private-PGM library provides a straightforward interface that allows users to quickly write end-to-end code to generate synthetic data; different statistics can be preserved by changing Line 6 .

\section{Overview of Measurement and Inference with Private-PGM}

In this section, we elaborate on the general template for a mechanism outlined in Figure 1. Recall there are three high-level steps:

(1) Select. Select a collection of marginals to measure.

(2) Measure. Use the Gaussian mechanism to measure each marginal in the collection.

(3) Generate. Use Private-PGM to estimate a data distribution from the noisy measurements and generate synthetic data that preserves the measured marginals well.

In this section, we describe the latter two steps, which form the core of the mechanism. In the next section, we will describe the full mechanism NIST-MST in detail, including the select step and many other details relating specifically to the NIST contest and dataset. Figure 2 shows how simple and modular this framework for synthetic data generation is. The open source Private-PGM library $[41]^{1}$ provides a simple interface to the key routines so that an end-to-end synthetic data generation mechanism can be written with very little code, allowing the modeler to focus on tailoring the procedure to the workload and domain. Under the hood, Private-PGM has thousands of lines of code, but it exposes a simple interface that is easy to use. In this example, there are only two selected marginal queries: (SEX, LABFORCE) and (LABFORCE, SCHOOL), but the code can be readily modified (Line 6) to accommodate other marginal queries. In the rest of the section, we describe this general approach in more detail, and give some insight into the steps described in this code snippet.

4.1. Measuring Marginals with the Gaussian Mechanism. Algorithm 1 shows the method for measuring marginals. Given a collection of attribute subsets $\mathcal{C}$, it computes the marginal for each $C \in \mathcal{C}$ and adds i.i.d. Gaussian noise to preserve privacy. It also accepts a weight $w_{C}$ for each attribute subset, which represents the relative importance of

\footnotetext{
${ }^{1}$ See also https://github.com/ryan112358/private-pgm/
} 


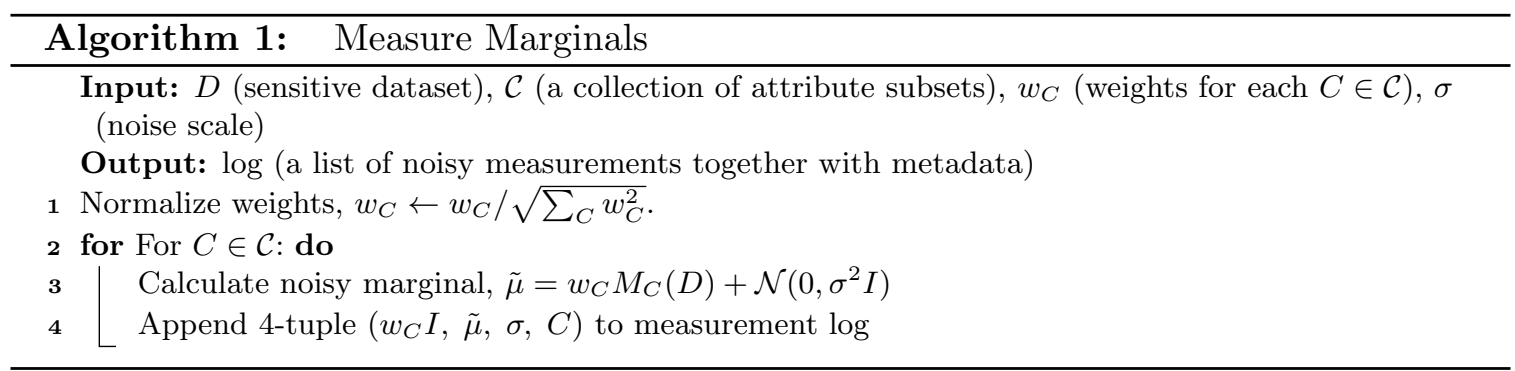

that marginal. It collects all of these noisy measurements into a measurement log, which will be passed to Private-PGM for post-processing. The measurement log records, for each marginal defined by a subset of attributes, the noisy marginal query answers together with information about the weight assigned to the marginal and the magnitude of noise used to measure it. It is easy to verify the privacy properties of Algorithm 1, as it is a direct application of the Gaussian mechanism on a sensitivity-1 quantity. ${ }^{2}$

Theorem 1. Algorithm 1 satisfies $\left(\alpha, \frac{\alpha}{2 \sigma^{2}}\right)-R D P$ for all $\alpha \geq 1$.

4.2. Private-PGM: Inference and Synthetic Data Generation. Private-PGM is a generalpurpose post-processing tool to infer a data distribution given noisy measurements [42]. It is compatible with measurements from a wide variety of mechanisms for discrete data, and can often improve utility at no cost to privacy. Because it infers a representation of a full data distribution, it produces query answers that are consistent with one another, even if the noisy measurements are inconsistent. It uses a compact representation of the data distribution to avoid exponential complexity in many cases, though the size of the representation will depend on the measurements, as we describe below.

The high-level idea of Private-PGM is to solve an optimization problem to find a data distribution that would produce measurements close to the ones that were observed. It applies to cases when private measurements depend on the data through marginals. For example, suppose the measurements are of the form

$$
y_{C}=Q_{C} M_{C}(D)+\xi
$$

for all attributes sets $C$ in some collection $\mathcal{C}$, where $Q_{C} \in \mathbb{R}^{p_{C} \times n_{C}}$ is a linear transformation applied to the marginal prior to release and $\xi \in \mathbb{R}^{p_{C}}$ is zero-centered noise (e.g., Laplace or Gaussian) with known standard deviation. The measurements taken in Algorithm 1 represent the common case where $Q_{C}$ is just the identity matrix, so that we observe the noisy marginals directly. However, the ability to measure arbitrary linear transformations of marginals is a nice feature that is useful for some types of measurements that occur in practice. ${ }^{3}$ Examples of this include hierarchical measurements for answering range queries [28,34,47], and optimized measurements for answering general linear query workloads $[35,40]$.

Given these measurements, Private-PGM infers a data distribution $P$ that best explains the measurements by solving the optimization problem

$$
\underset{P}{\operatorname{argmin}} \sum_{C \in \mathcal{C}}\left\|Q_{C} M_{C}(P)-y_{C}\right\|_{2}^{2} .
$$

\footnotetext{
${ }^{2}$ The weights are explicitly normalized so that the collection of marginals has sensitivity 1.

${ }^{3}$ In fact, $Q_{C}$ can be replaced with an arbitrary non-linear differentiable transformation, and Private-PGM will accept that as input as well.
} 
The objective of this optimization problem is the negative log-likelihood of the noisy measurements under the Gaussian release mechanism, so Equation (4.1) can be seen as a maximum likelihood estimator. We have abused notation by allowing $M_{C}$ to operate on a data distribution rather than a dataset; the correct interpretation is to substitute the probability vector $P$ for the contingency table representation $D$ of the dataset for computing the marginal. An obvious issue with the optimization problem in Equation (4.1) is that the dimension of the decision variable $P$ is equal to the domain size $n$, which is exponential in the number of attributes, so we cannot usually solve this problem directly. The key observation of Private-PGM is

Fact 1. Equation (4.1) has an optimum of the form $P_{\theta}$, where $P_{\theta}$ is a graphical model with one factor for each set $C \in \mathcal{C}$ of attributes for which the mechanism measured a marginal.

This allows us to solve the much lower-dimensional optimization problem

$$
\underset{\theta}{\operatorname{argmin}} \sum_{C \in \mathcal{C}}\left\|Q_{C} M_{C}\left(P_{\theta}\right)-y_{C}\right\|_{2}^{2}
$$

with no loss in solution quality. The decision variable $\theta$ is the parameter vector of the graphical model, and has dimension equal to the total length of the set of measured marginals. A simple proximal algorithm is given in [42] that solves this optimization problem using only repeated calls to a routine to perform marginal inference in a discrete graphical model - i.e., computing $M_{C}\left(P_{\theta}\right)$ for all $C \in \mathcal{C}$ and various different $\theta$. The procedure is efficient whenever marginal inference in the graphical model is efficient. Belief propagation is the standard way to perform marginal inference in practice, as it efficiently computes $M_{C}\left(P_{\theta}\right)$ directly in terms of $\theta$ without ever explicitly materializing the full joint distribution $P_{\theta}$ [32].

Remark 1 Scalability of Private-PGM. Private-PGM is able to scale to very high-dimensional domains. The main factors that influence it's scalability are (1) the total size of the parameter vector $\theta$ and (2) the structure of the set $\mathcal{C}$. The size of $\theta$ is the same as the size of all of the relevant marginals combined, and that must not be too large. The size of each marginal depends directly on $\left|\Omega_{i}\right|$, the number of possible values for each attribute. Furthermore, the set $\mathcal{C}$ is important because it corresponds to the structure of the graphical model, and belief propagation is most efficient for tree-structured models. The scalability of belief propagation and Private-PGM for non tree-structured models depends on a quantity known as the tree width, which is a measure of how "tree-like" the model is [32].

Remark 2 Lack of modeling assumptions. It is easy to misconstrue the meaning of the graphical model representation. The inferred distribution $P_{\theta}$ will satisfy conditional independence properties dictated by the structure of the model. However, no approximation is made when solving the optimization problem in Equation (4.1), and the independence properties do not arise from assumptions made by the modeler about the structure of the data distribution. By Fact 1, there is an optimum to Equation (4.1) that is a graphical model, and hence satisfies these conditional independence properties. Moreover, the graphical model solution $P_{\theta}$ can be shown to have maximum entropy among all optima of Equation (4.1) [42].

Once $P_{\theta}$ is estimated, Private-PGM can be used for multiple purposes: reducing error on measured marginals, estimating unmeasured marginals, and even generating synthetic tabular data. These use cases are explained in greater detail below: 
Reducing error on measured marginals. First, Private-PGM improves utility by combining all sources of measured information into a single cohesive estimate for the data distribution. When the measurements are inconsistent with each other, Private-PGM resolves these inconsistencies in a principled manner, reducing variance and boosting utility. One achieves this by using $\bar{y}_{C}=Q_{C} M_{C}\left(P_{\theta}\right)$ in place of the noisy observation $y_{C}$. The estimated marginal $\bar{y}_{C}$ will typically have smaller variance than $y_{C}$ and will often have lower overall error as well, therefore offering immediate utility improvements at no cost to privacy. Example 1 demonstrates this idea more concretely in a toy setting.

Example 1 (Boosting utility on measured marginals). We draw 1000 tuples from the actual contest dataset and measure two of their marginals, (SEX, LABFORCE) and (LABFORCE, SCHOOL), using the Gaussian mechanism with $\sigma=50$. Tables $(a-c)$ below show the true marginals, the noisy marginals, and the marginals estimated by Private-PGM. One can easily verify that the noisy marginals are not consistent: the (SEX, LABFORCE) marginal implies the total number of people with $L A B F O R C E=N$ is $124.549+318.029=442.578$, while the (LABFORCE, SCHOOL) marginal implies that the same number is $287.215+171.134=458.349$. These are two different estimates for the same quantity, which is a consistency problem. In contrast, the Private-PGM estimated marginals are consistent: they both agree that the total number is 436.873. Additionally, Private-PGM better estimates the true marginals than the noisy marginals do: the $L_{1}$ distances are about 213 and 272 for Private-PGM, while they are about 251 and 295 for the noisy marginals, which is a significant boost in utility.

\begin{tabular}{cc|c}
\hline SEX & LABFORCE & count \\
\hline$M$ & - & 156 \\
$M$ & $N$ & 65 \\
$M$ & $Y$ & 316 \\
$F$ & - & 158 \\
$F$ & $N$ & 282 \\
$F$ & $Y$ & 23 \\
\hline \hline LABFORCE & $S C H O O L$ & count \\
\hline- & $N$ & 159 \\
- & $Y$ & 155 \\
$N$ & $N$ & 288 \\
$N$ & $Y$ & 59 \\
$Y$ & $N$ & 336 \\
$Y$ & $Y$ & 3 \\
\hline
\end{tabular}

(a) True marginals

\begin{tabular}{cc|c}
\hline SEX & LABFORCE & count \\
\hline$M$ & - & 132.428 \\
$M$ & $N$ & 124.549 \\
$M$ & $Y$ & 244.365 \\
$F$ & - & 173.633 \\
$F$ & $N$ & 318.029 \\
$F$ & $Y$ & -21.358 \\
\hline \hline LABFORCE & $S C H O O L$ & count \\
\hline- & $N$ & 116.021 \\
- & $Y$ & 186.826 \\
$N$ & $N$ & 287.215 \\
$N$ & $Y$ & 171.134 \\
$Y$ & $N$ & 278.498 \\
$Y$ & $Y$ & -46.497 \\
\hline
\end{tabular}

(b) Noisy marginals

\begin{tabular}{cc|c}
\hline$S E X$ & LABFORCE & count \\
\hline$M$ & - & 124.829 \\
$M$ & $N$ & 121.696 \\
$M$ & $Y$ & 254.636 \\
$F$ & - & 166.034 \\
$F$ & $N$ & 315.177 \\
$F$ & $Y$ & 0 \\
\hline \hline LABFORCE & $S C H O O L$ & count \\
\hline- & $N$ & 110.029 \\
- & $Y$ & 180.834 \\
$N$ & $N$ & 276.477 \\
$N$ & $Y$ & 160.396 \\
$Y$ & $N$ & 254.636 \\
$Y$ & $Y$ & 0 \\
\hline
\end{tabular}

(c) Private-PGM marginals

Estimating unmeasured marginals. Second, Private-PGM can be used to answer new queries that were never measured directly by using $P_{\theta}$ in place of the true data $D$. This allows us to estimate new marginals without spending the privacy budget, saving a precious resource. Example 2 demonstrates this idea in a toy setting.

Example 2 (Estimating new marginals). Building on Example 1, recall that we measured the marginals on (SEX, LABFORCE) and (LABFORCE, SCHOOL). We can use Private-PGM to estimate the marginal on (SEX,SCHOOL), even though we never measured it and it can not be directly inferred from the other marginals that were measured. As shown below in Table (b), the provided estimate is reasonable, given that we never measured it, and we added significant noise to the marginals we did measure. We reiterate that we obtained this estimate "for free", without spending additional privacy budget. Additionally, Private-PGM can estimate 
the marginal on (SEX, LABFORCE, SCHOOL), which is shown in Table (d). This is pretty close to the true 3-way marginal, shown in Table (c). In fact, the normalized $L_{1}$ error is only 0.135. While there may be other equally good estimates for the 3-way marginal (according to the loss function in Equation (4.1)), the estimate provided by Private-PGM has maximum entropy among all of them. In this case, Private-PGM was fairly accurate because SEX and SCHOOL are (approximately) conditionally independent given LABFORCE in the true data.

\begin{tabular}{cc|r}
\hline SEX & SCHOOL & count \\
\hline$M$ & $N$ & 423 \\
$M$ & $Y$ & 114 \\
$F$ & $N$ & 360 \\
$F$ & $Y$ & 103
\end{tabular}

(a) True 2-way marginal

\begin{tabular}{cc|c}
\hline SEX & SCHOOL & count \\
\hline$M$ & $N$ & 378.873 \\
$M$ & $Y$ & 122.289 \\
$F$ & $N$ & 262.269 \\
$F$ & $Y$ & 218.942
\end{tabular}

(b) Estimated 2-way marginal

\begin{tabular}{ccc|r}
\hline SEX & LABFORCE & SCHOOL & count \\
\hline$M$ & - & $N$ & 74 \\
$M$ & - & $Y$ & 82 \\
$M$ & $N$ & $N$ & 36 \\
$M$ & $N$ & $Y$ & 29 \\
$M$ & $Y$ & $N$ & 313 \\
$M$ & $Y$ & $Y$ & 3 \\
$F$ & - & $N$ & 85 \\
$F$ & - & $Y$ & 73 \\
$F$ & $N$ & $N$ & 252 \\
$F$ & $N$ & $Y$ & 30 \\
$F$ & $Y$ & $N$ & 23 \\
$F$ & $Y$ & $Y$ & 0
\end{tabular}

(c) True 3-way marginal

\begin{tabular}{ccc|r}
\hline SEX & LABFORCE & SCHOOL & \multicolumn{1}{c}{ count } \\
\hline$M$ & - & $N$ & 47.221 \\
$M$ & - & $Y$ & 77.608 \\
$M$ & $N$ & $N$ & 77.016 \\
$M$ & $N$ & $Y$ & 44.68 \\
$M$ & $Y$ & $N$ & 254.636 \\
$M$ & $Y$ & $Y$ & 0.000 \\
$F$ & - & $N$ & 62.808 \\
$F$ & - & $Y$ & 103.226 \\
$F$ & $N$ & $N$ & 199.461 \\
$F$ & $N$ & $Y$ & 115.716 \\
$F$ & $Y$ & $N$ & 0.000 \\
$F$ & $Y$ & $Y$ & 0.000
\end{tabular}

(d) Estimated 3-way marginal

Generating synthetic data. Third, Private-PGM can be used to generate synthetic data $\bar{D}$ in tabular format. $\bar{D}$ can be used in place of $P_{\theta}$ and will generally give similar results. They will not give exactly the same results because $\bar{D}$ has integer-valued marginals while $P_{\theta}$ has real-valued marginals, so some additional rounding error is unavoidable. One can obtain the synthetic data in multiple ways; a simple and natural approach would be to sample records from $P_{\theta}$ to form a synthetic dataset. This naive approach would introduce sampling error which is undesirable. Private-PGM uses an alternative approach to reduce error from additional sources of randomness. The details of this procedure are available in the open-source implementation of Private-PGM, and are summarized in the supplementary material. In Example 3, we give an intuitive idea of how this procedure works, and illustrate why it is preferable to the sampling approach.

Example 3 (Generating synthetic data). Building on Examples 1 and 2, we calculate the LABFORCE marginal from the Private-PGM model in Table (a) below, which has fractional counts. We also use Private-PGM to generate synthetic data and show the same marginal in Table (b), which has integer counts. These two marginals almost exactly match, because Private-PGM tries to preserve the model marginals as closely as possible when generating synthetic data. However, synthetic data obtained by i.i.d sampling will not match the model marginals as closely due to the randomness in sampling, as shown in Table (c).

\begin{tabular}{c|c}
\hline LABFORCE & count \\
\hline- & 290.863 \\
$N$ & 436.873 \\
$Y$ & 254.636
\end{tabular}

(a) Private-PGM model marginal

\begin{tabular}{c|c}
\hline LABFORCE & count \\
\hline- & 291 \\
$N$ & 437 \\
$Y$ & 254
\end{tabular}

(b) Private-PGM synthetic data marginal

\begin{tabular}{c|c}
\hline LABFORCE & count \\
\hline- & 262 \\
$N$ & 468 \\
$Y$ & 252
\end{tabular}

(c) Sampled synthetic data marginal 


\begin{tabular}{|c|c|c|}
\hline \multicolumn{3}{|c|}{ Algorithm 2: NIST-MST } \\
\hline 1 Calibrate Noise & Derive noise scale $\sigma$ from target privacy parameters $(\epsilon, \delta)$ & Equation (5.1) \\
\hline 2 Encode Domain & Use public information to encode attribute domains & \\
\hline 3 Transform Data & Transform data using insights from provisional data & Algorithm 3 \\
\hline 4 Compress Domain & Use data to reduce domain: & \\
\hline Measure & Measure all one-way marginals & Algorithm 1 \\
\hline Compress & Remove domain elements failing threshold test & Algorithm 4 \\
\hline 5 Select Marginals & Select a subset of 2- and 3-way marginals & Algorithm 5 \\
\hline 6 Measure Marginals & Measure selected marginals & Algorithm 1 \\
\hline 7 Synthesize data & Synthesize records using Private-PGM: & \\
\hline Estimate & Estimate distribution from Step 4 and 6 measurements & Equation (4.2) \\
\hline Generate & Generate synthetic records & Algorithm 8 \\
\hline 8 Reverse & Reverse the transformation made in Step 3 & Algorithm 9 \\
\hline
\end{tabular}

\section{Algorithm Description}

In this section we describe NIST-MST, which takes the basic mechanism template outlined in the previous section, and applies it to the setting of the NIST competition. NIST-MST simply invokes the Gaussian mechanism to measure a carefully chosen subset of 1, 2, and 3-way marginals. Then the resulting noisy measurements are post-processed using Private-PGM to obtain synthetic data that is most consistent with those marginals. NIST-MST does not follow the template from the previous section exactly, as there are two rounds of measurements, and an additional domain compression step developed specifically to deal with some of the challenges around the dataset used in the competition. The high-level steps of NIST-MST are stated in Algorithm 2, and a detailed description of each step will be provided in this section, with motivations and intuitions for the various design choices.

Step 1: Calibrate Noise. In this step, $\sigma$ is calibrated to ensure the whole algorithm satisfies $(\epsilon, \delta)$-differential privacy. Note that only steps (4) and (6) in Algorithm 2 use the sensitive data, and these are both invocations of Algorithm 1, which is $\left(\alpha, \frac{\alpha}{2 \sigma^{2}}\right)$-RDP (Theorem 1). The data transformations made in steps (3) and (4) do not affect the privacy analysis of Algorithm 1 since one individual can still only affect each marginal by at most one. Hence NIST-MST is $\left(\alpha, \frac{\alpha}{\sigma^{2}}\right)$-RDP by two-fold adaptive composition (Proposition 3 ). Moreover, by Proposition 4, NIST-MST is $\left(\frac{\alpha}{\sigma^{2}}+\frac{\log (1 / \delta)}{\alpha-1}, \delta\right)$-DP for all $\alpha \geq 1$.

For a fixed $\alpha$, it is easy to determine $\sigma$ by solving the equation $\frac{\alpha}{\sigma^{2}}+\frac{\log (1 / \delta)}{\alpha-1}=\epsilon$ for $\sigma$. The best value of $\sigma$ can be obtained by minimizing over all $\alpha$. In the contest, this computation was done by invoking the moments accountant [1], which minimizes over $\alpha=1, \ldots, 512$. However, this minimization can actually be done in closed form [73], leading to the following equation for $\sigma$ :

$$
\sigma=\frac{\sqrt{\log (1 / \delta)}+\sqrt{\log (1 / \delta)+\epsilon}}{\epsilon}
$$

Theorem 2 (Privacy of NIST-MST). Algorithm 2 is $(\epsilon, \delta)$-differentially private.

Proof. From the analysis above, we know that NIST-MST is $\left(\frac{\alpha}{\sigma^{2}}+\frac{\log (1 / \delta)}{\alpha-1}, \delta\right)$-DP for all $\alpha \geq 1$. By plugging in $\alpha=1+\sigma \sqrt{\log (1 / \delta)}$ and $\sigma=\frac{\sqrt{\log (1 / \delta)}+\sqrt{\log (1 / \delta)+\epsilon}}{\epsilon}$ and simplifying, we see that $\frac{\alpha}{\sigma^{2}}+\frac{\log (1 / \delta)}{\alpha-1}=\epsilon$, and hence NIST-MST is $(\epsilon, \delta)$-DP as desired. The algebraic 


\begin{tabular}{|c|c|c|c|c|c|c|c|c|c|}
\hline 1. SPLIT & $2 / 2$ & 21. NCHLT5 & $7 / 7$ & 41. SIZEPL & $31 / 19$ & 61. SUPDIST & $631 / 631$ & 81. ENUMDIST & $3021 / 3021$ \\
\hline 2. SLREC & $3 / 2$ & 22. RACE & $7 / 7$ & 42. EMPSTATD & $35 / 15$ & 62. METAREA & $657 / 334$ & 82. CITYPOP & $3225 / 3225$ \\
\hline 3. SEX & $3 / 2$ & 23. WKSWORK2 & $7 / 7$ & 43. WKSWORK1 & $53 / 53$ & 63. PRESGL & $816 / 816$ & 83. URBPOP & $3225 / 3225$ \\
\hline 4. SCHOOL & $3 / 2$ & 24. VET1940 & $9 / 4$ & 44. SEA & $54 / 54$ & 64. BPL & $901 / 163$ & 84. METAREAD & $6561 / 378$ \\
\hline 5. URBAN & $3 / 3$ & 25. UCLASSWK & $9 / 8$ & 45. OCCSCORE & $81 / 81$ & 65. MBPL & $901 / 164$ & 85. MTONGUED & $9602 / 489$ \\
\hline 6. FARM & $3 / 3$ & 26. VETPER & $9 / 8$ & 46. AGEMARR & $90 / 89$ & 66. FBPL & $901 / 165$ & 86. MIGMET5 & $10000 / 379$ \\
\hline 7. OWNERSHP & $3 / 3$ & 27. HISPRULE & $9 / 9$ & 47. MIGRATE5D & $91 / 15$ & 67. IND1950 & $998 / 162$ & 87. MIGCOUNTY & $10000 / 385$ \\
\hline 8. RESPONDT & $3 / 3$ & 28. HRSWORK2 & $9 / 9$ & 48. MTONGUE & $97 / 92$ & 68. MIGSEA5 & $998 / 510$ & 88. ERSCOR50 & $10000 / 1002$ \\
\hline 9. SPANNAME & $3 / 3$ & 29. CLASSWKR & $10 / 4$ & 49. SEI & $97 / 97$ & 69. OCC & $999 / 231$ & 89. EDSCOR50 & $10000 / 1002$ \\
\hline 10. LABFORCE & $3 / 3$ & 30. INCNONWG & $10 / 4$ & 50. CLASSWKRD & $99 / 18$ & 70. EDUCD & $1000 / 44$ & 90. NPBOSS50 & $10000 / 1002$ \\
\hline 11. VETWWI & $3 / 3$ & 31. SAMEPLAC & $10 / 4$ & 51. HRSWORK1 & $99 / 99$ & 71. HIGRADED & $1000 / 69$ & 91. CITY & $10000 / 1164$ \\
\hline 12. SSENROLL & $3 / 3$ & 32. VETSTAT & $10 / 4$ & 52. VETSTATD & $100 / 10$ & 72. GQTYPED & $1000 / 92$ & 92. MIGCITY5 & $10000 / 1164$ \\
\hline 13. METRO & $4 / 4$ & 33. VETCHILD & $10 / 5$ & 53. GQFUNDS & $100 / 13$ & 73. IND & $1000 / 136$ & 93. RENT & $10000 / 10000$ \\
\hline 14. EMPSTAT & $4 / 4$ & 34. MIGTYPE5 & $10 / 6$ & 54. EDUC & $100 / 13$ & 74. UIND & $1000 / 136$ & 94. MBPLD & $90021 / 537$ \\
\hline 15. HISPAN & $5 / 5$ & 35. SAMESEA5 & $10 / 6$ & 55. AGEMONTH & $100 / 15$ & 75. MIGPLAC5 & $1000 / 199$ & 95. FBPLD & $90021 / 539$ \\
\hline 16. CITIZEN & $5 / 5$ & 36. MIGRATE5 & $10 / 7$ & 56. HIGRADE & $100 / 25$ & 76. UOCC & $1000 / 231$ & 96. BPLD & $90022 / 536$ \\
\hline 17. WARD & $6 / 6$ & 37. GQTYPE & $10 / 10$ & 57. CHBORN & $100 / 62$ & 77. UOCC95 & $1000 / 279$ & 97. VALUEH & $10000000 / 5003$ \\
\hline 18. NATIVITY & $6 / 6$ & 38. MARRNO & $10 / 10$ & 58. AGE & $109 / 109$ & 78. 0CC1950 & $1000 / 283$ & 98. INCWAGE & $-/ 52$ \\
\hline 19. MARST & $7 / 6$ & 39. OWNERSHPD & $21 / 8$ & 59. HISPAND & $481 / 55$ & 79. DURUNEMP & $1000 / 1000$ & 99. INCWAGE $E_{B}$ & $-/ 8$ \\
\hline 20. GQ & $7 / 7$ & 40. FAMSIZE & $22 / 22$ & 60. RACED & $621 / 238$ & 80. COUNTY & $1251 / 385$ & INCWAGE & $10000000 /-$ \\
\hline
\end{tabular}

Table 4. Domain information for the census dataset used in the third round of the competition. Table specifies attribute names, and number of possible values for that attribute according to (1) the provided specs file and (2) the specs file combined with IPUMS documentation.

manipulation is routine but messy; it can easily be verified with sympy (see Figure 4 in the supplement).

Remark 3 Noise Calibration. It is well known that calibrating $\sigma$ via an RDP analysis does not give the smallest possible value required to achieve $(\epsilon, \delta)-D P$, and an analytic calibration gives strictly better results [6], at least for a single invocation of the Gaussian mechanism. However, at the time of the competition, adaptive composition of two Gaussian mechanisms was needed, and RDP was chosen because of its clean and well-understood guarantees. If using the analytic Gaussian mechanism, advanced composition would be necessary to reason about the privacy of two-fold adaptive composition [20]. Since these are somewhat loose bounds, the benefit of the analytic calibration would be lost. However, since the time of the competition, much progress has been made on understanding the behavior of the Gaussian mechanism under composition and it is now known that the analytic Gaussian mechanism can be used to calibrate noise for multiple (adaptive) invocations of the Gaussian mechanism [18, 52]. This would give a smaller value of $\sigma$ than the one shown in Equation (5.1), typically offering an improvement of 10 to 20 percent.

Step 2: Encode Domain. Before running NIST-MST, it is necessary to know the data domain $\Omega$. The supplied competitor pack came with a "SPECS" file that contained some domain information. Specifically, it supplied a single positive integer $n_{i}$ for each attribute $i$, and the domain for that attribute was assumed to be $\Omega_{i}^{\text {SPECS }}=\left\{0, \ldots, n_{i}-1\right\}$. However, because the data is derived from a census source, the domain is very thoroughly documented on the Integrated Public Use Microdata Series (IPUMS) website [49]. IPUMS offers a much finer grained view of the data domain, specifying the exact set of possible values for most attributes. We use $\Omega_{i}^{\text {IPUMS }}$ to denote the domain of possible values for attribute $i$ according to IPUMS. Example 4 demonstrates the benefit of using the finer grained IPUMS domain information. 


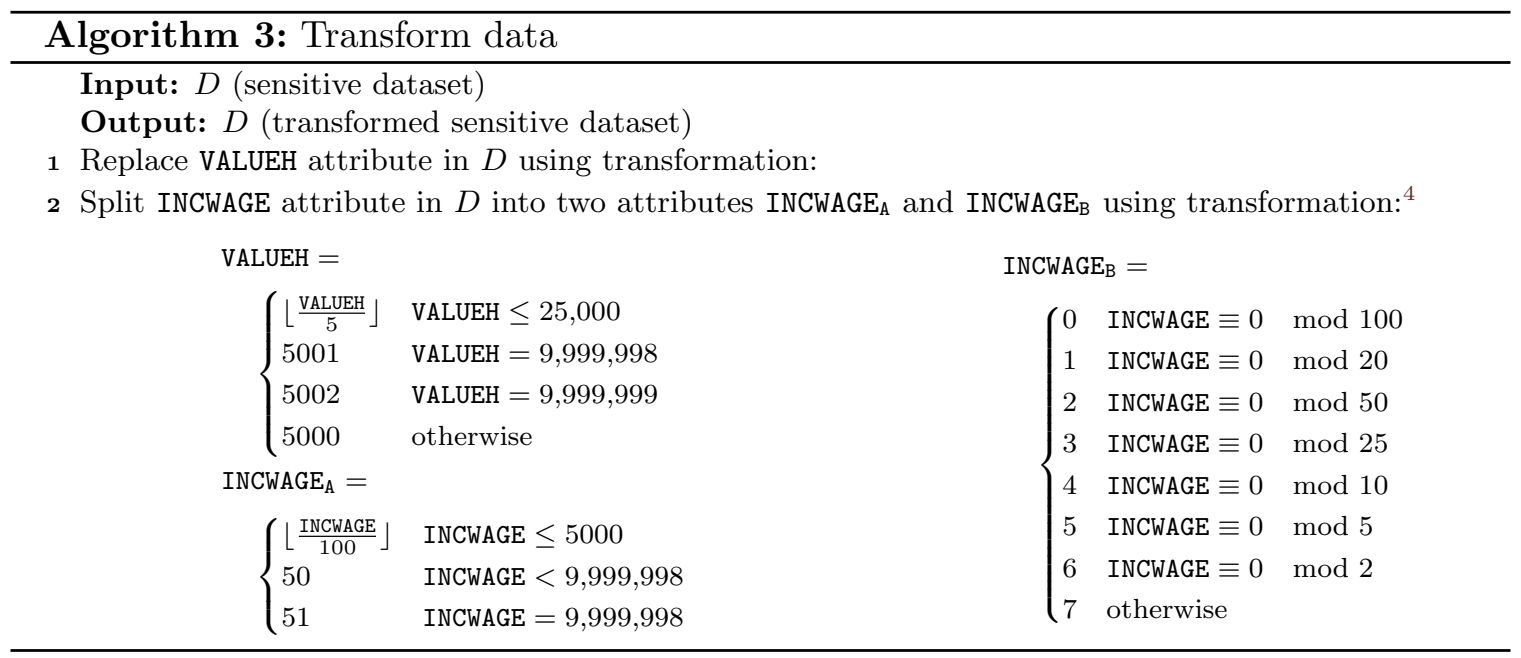

Example 4 (SPECS vs. IPUMS). From the specs file and the IPUMS website, we see the domain for the EDUC attribute is $\Omega_{i}^{S P E C S}=\{0,1, \ldots, 99\}$ and $\Omega_{i}^{I P U M S}=\{0,1, \ldots, 11,99\}$. Note that 99 is a special code that typically corresponds to missing data. While both sources agree that 99 is the largest possible value, the IPUMS documentation suggests that values in the range $12, \ldots, 98$ are not possible. Using the finer granularity domain from IPUMS reduces the number of possible values for EDUC from 100 to 13. This has two important ramifications. First, it will make Private-PGM more efficient in later steps, since the scalability of that tool depends directly on the domain sizes of the attributes. Second, it will prevent NIST-MST from inadvertently introducing out-of-domain tuples to the synthetic data which could otherwise occur by adding positive noise to zero counts.

Often $\Omega_{i}^{\text {IPUMS }}$ is a subset of $\Omega_{i}^{\text {SPECS }}$, although this is not always the case. In some cases, IPUMS documents a certain value as being possible that never appeared in the provisional dataset or the supplied specs file. To account for this NIST-MST uses the intersection of the two domains, i.e., $\Omega_{i}=\Omega_{i}^{\text {SPECS }} \cap \Omega_{i}^{\text {IPUMS }}$. Table 4 enumerates the attributes in the dataset along with the domain size provided in the specs file, and the compressed domain size derived by NIST-MST.

Step 3: Transform Data. In addition to the general domain encoding outlined above, NIST-MST gave special attention to two of the attributes with the largest domain: INCWAGE and VALUEH. Both of these attributes started out with 10 million possible values, and the IPUMS documentation provided limited information on these attributes. Therefore, NIST-MST leveraged the provisional dataset to try to identify a domain that captured all or most of the observed values for these attributes. Algorithm 3 shows how these attributes are transformed to reduce the domain size. The intuition behind this pre-processing procedure is to compress the domain, while ensuring the compressed domain still covers all or most of the values observed in the provisional dataset. For example, other than the special codes of $9,999,998$ and $9,999,999,99.2 \%$ of records have VALUEH that is a multiple of 5 and less

\footnotetext{
${ }^{4}$ Each condition in the piecewise definition of INCWAGE and INCWAGE should be interpreted as an "else if" statement rather than an "if" statement, as clearly multiple conditions can be true at the same time
} 


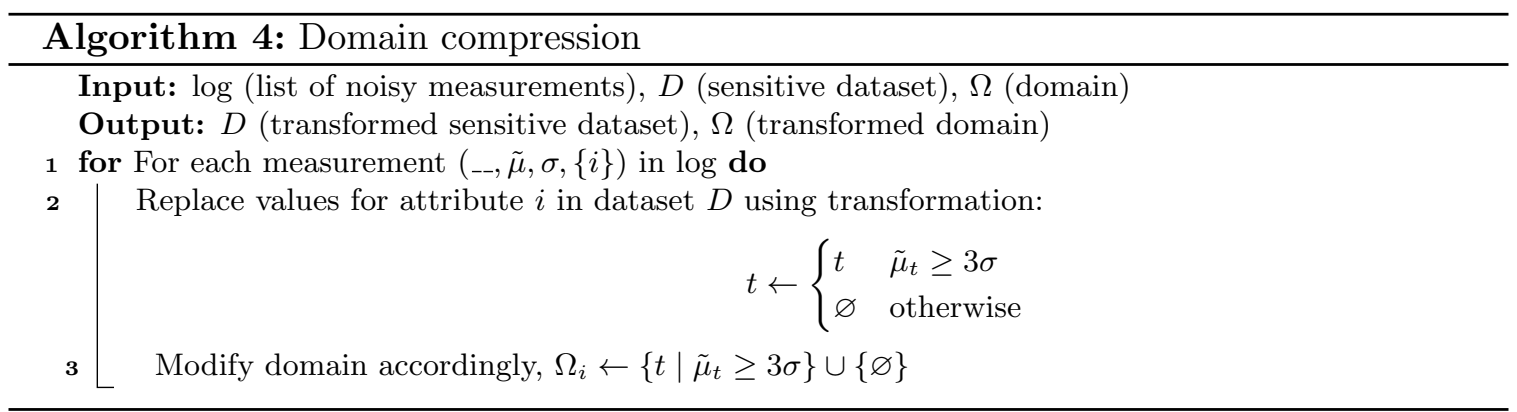

than or equal to $25,000 .^{5}$ This allows us to compress the domain of VALUEH to a much more manageable size of 5003 while still covering about $99.7 \%$ of the observed values.

NIST-MST uses a similar approach to handle INCWAGE. Over $99.95 \%$ of records in the provisional dataset had an INCWAGE value of either $9,999,998$ or something in the range $[0,5000]$. For that reason, it is reasonably safe to truncate values above 5000 without introducing too much bias. This transformation reduces the domain size of INCWAGE to 5002, but NIST-MST takes things one step further. There are clear periodic patterns in the INCWAGE marginal, as the most common values are all multiples of 100. Multiples of 20, 50, and 25 are also common. To exploit this observation, NIST-MST splits up INCWAGE into two attributes: INCWAGE $_{A}$ and INCWAGE $E_{B}$, and never measures INCWAGE directly, but only indirectly through these two derived attributes. INCWAGE $\mathrm{A}_{\mathrm{A}}$ is meant to capture the coarse-grained income by discretizing it into width 100 bins, whereas INCWAGE $_{B}$ is meant to capture the periodicity in the last two digits. These two derived attributes have smaller domains of size 52 and 8 , respectively. The exact formulas are given in Algorithm 3.

Step 4: Compress Domain. In step (1), NIST-MST was able to greatly reduce the domain size by incorporating information from IPUMS. However, even after this domain encoding, some of the attributes in the data remain fairly sparse. For example, only $17.4 \%$ percent of counts in the VALUEH marginal exceed 100. In this step, we answer all 1-way marginals, i.e., we pass $\mathcal{C}=\{\{i\} \mid i \in \mathcal{A}\}$ into Algorithm 1. Every marginal is assigned an equal weight of 1 , with the exception of $\operatorname{INCWAGE}_{\mathrm{A}}$, which is assigned a weight of 2.

After obtaining noisy 1-way marginals, Algorithm 4 is called, which searches for domain elements for which the noisy count fell below the threshold of $3 \sigma$. These domain elements were merged into a single "other" domain element, denoted $\varnothing$. Later steps of NIST-MST operate over the resulting transformed dataset and domain. This step has two main benefits, similar to the ones from the domain encoding step. First, it improves scalability of Private-PGM in later steps by reducing the domain size of the attributes. Second, it ensures that the tuples generated by NIST-MST (probably) have attribute values that actually occurred in the dataset.

Step 5: Select Measurements. The next step of NIST-MST is to identify a collection of 2and 3-way marginals that will later be measured. This is one of the most crucial components of NIST-MST, because the marginals selected in this step will ultimately determine the marginals that will be preserved in the generated synthetic data. It is important to note that this step selects measurements without using the sensitive dataset, although it does rely heavily on the provisional dataset. This algorithm does take the privacy budget $\epsilon$ as

\footnotetext{
${ }^{5} 9,999,998$ and $9,999,999$ are special codes corresponding to missing values of $\mathrm{N} / \mathrm{A}$ values.
} 


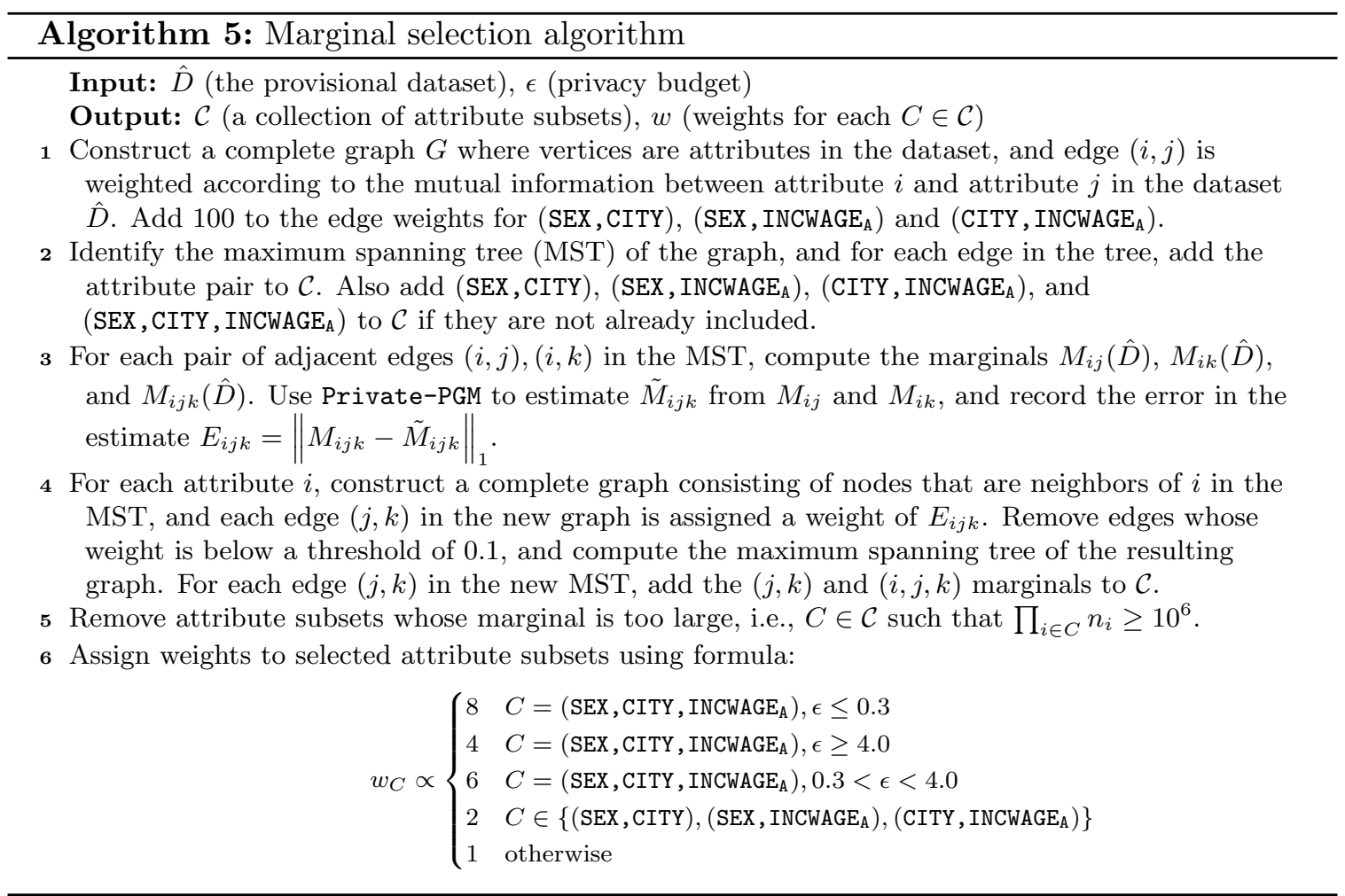

input, but it does not "consume" it — it only uses it to determine weights to assign to each selected marginal.

Algorithm 5 shows how NIST-MST selects 2- and 3-way marginals for measurement. This algorithm is inspired by a similar approach used by two other mechanisms for differentially private synthetic data [13,67]. It combines one principled step, which is to find the maximum spanning tree (MST) on the graph where edge weights correspond to mutual information between two attributes, with some additional heuristics to ensure that certain important attribute pairs are selected, and more heuristics to select some triples while keeping the graph tree-like. A reader familiar with graphical models will recognize the MST step as the famous Chow-Liu algorithm for structure learning in a graphical model [14]. Intuitively, highly correlated marginals should be measured because attributes that are independent can trivially be preserved without direct measurement.

Figure 3 shows the marginals selected by this algorithm in graphical format. Each edge in the graph represents a pair of attributes whose marginal will be measured by NIST-MST, and each triangle in the graph represents a triple of attributes whose marginal will be measured by NIST-MST. The structure of this measurement graph also corresponds to the structure of the graphical model used by Private-PGM. The tree-like structure of the graph will ultimately allow Private-PGM to run efficiently. The green subgraph corresponds to the (SEX, CITY, INCWAGE ${ }_{A}$ ) clique, the black edges form a maximum spanning tree of the underlying correlation graph, and the dotted red edges are additional edges that enhance the expressive capacity of the model while retaining the tree-like structure. 


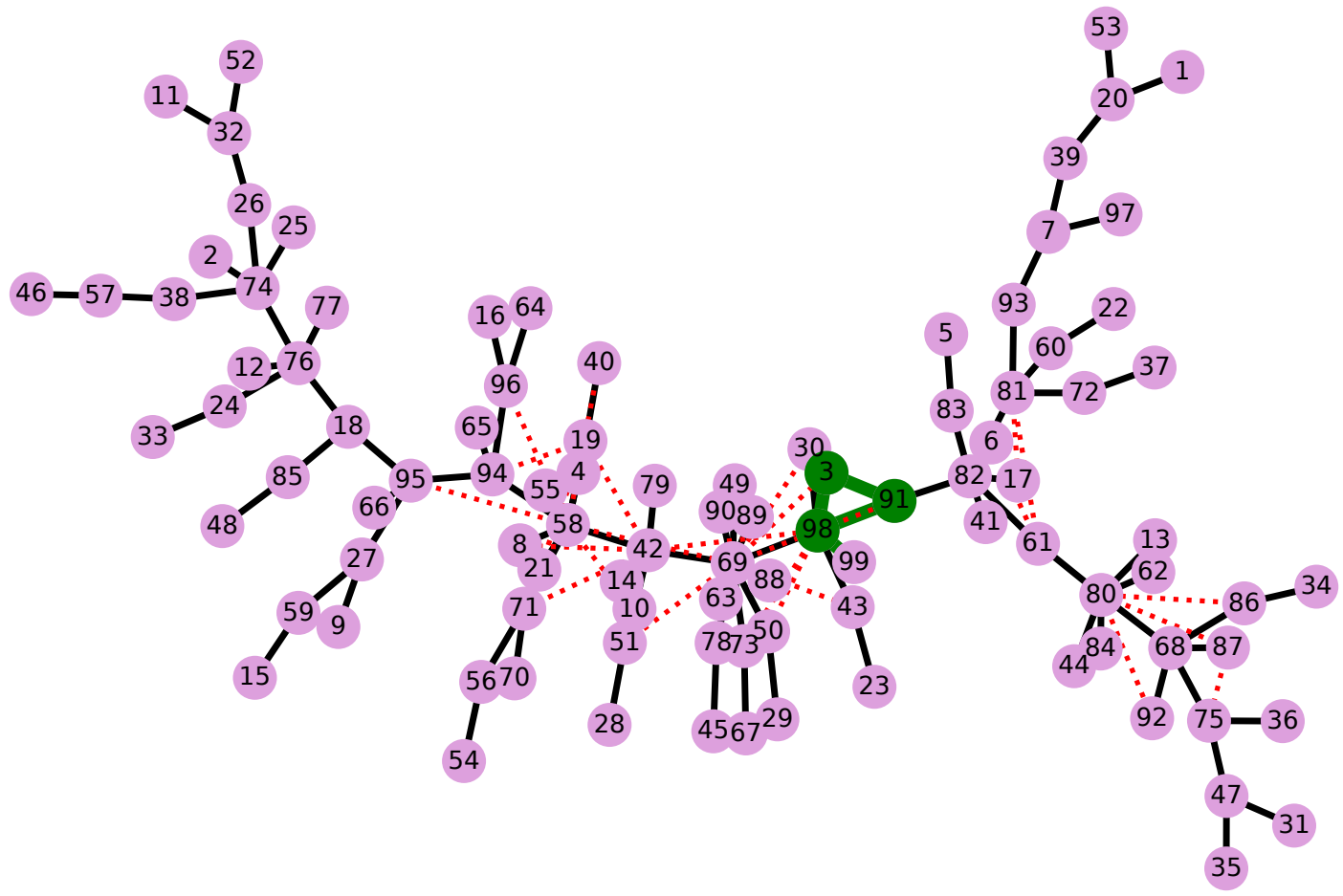

Figure 3. A graphical depiction of the 2- and 3-way marginals selected by NIST-MST. Nodes correspond to attributes in the dataset, and edges correspond to marginals selected by NIST-MST. Nodes are labeled by the 2-digit code for the attribute given in Table 4. Black edges form a maximum spanning tree of the underlying correlation graph. Green nodes and edges correspond to the special (SEX, CITY, INCWAGE $\mathrm{A}$ ) attributes. Dotted red edges identify the extra marginals chosen to improve expressive capacity of the model while maintaining tractability of Private-PGM. All dotted red edges form a triangle, and for each of those NIST-MST also included the 3-way marginal corresponding to the three nodes that make up the triangle in the list of selected marginals.

Step 6: Measure Marginals. The next step of NIST-MST is to measure the marginals selected in the previous step with Algorithm 1. The result is a collection of noisy measurements contained within a measurement log, and suitable for post-processing with Private-PGM.

Step 7: Synthesize data. The next step of NIST-MST is to combine the measurement logs from Steps 4 and 6 and pass them to Private-PGM, which returns a synthetic dataset whose marginals approximately match those in the measurement log. Because the measurements in Steps 4 and 6 were made on the uncompressed and compressed domains, respectively, the measurements from Step 4 had to be re-expressed over the compressed domain.

Step 8: Reverse Transformation. The final step of NIST-MST is to reverse the transformations made in Steps 4 and 3, to bring the data back to the original domain. For Step 4 , this requires evenly distributing any instances of $\varnothing$ among the original domain elements mapped to it. For Step 3, this requires modifying VALUEH and combining INCWAGE $A$ and INCWAGE $_{B}$ back into a single attribute. The details are given in Algorithm 9 of the appendix. 


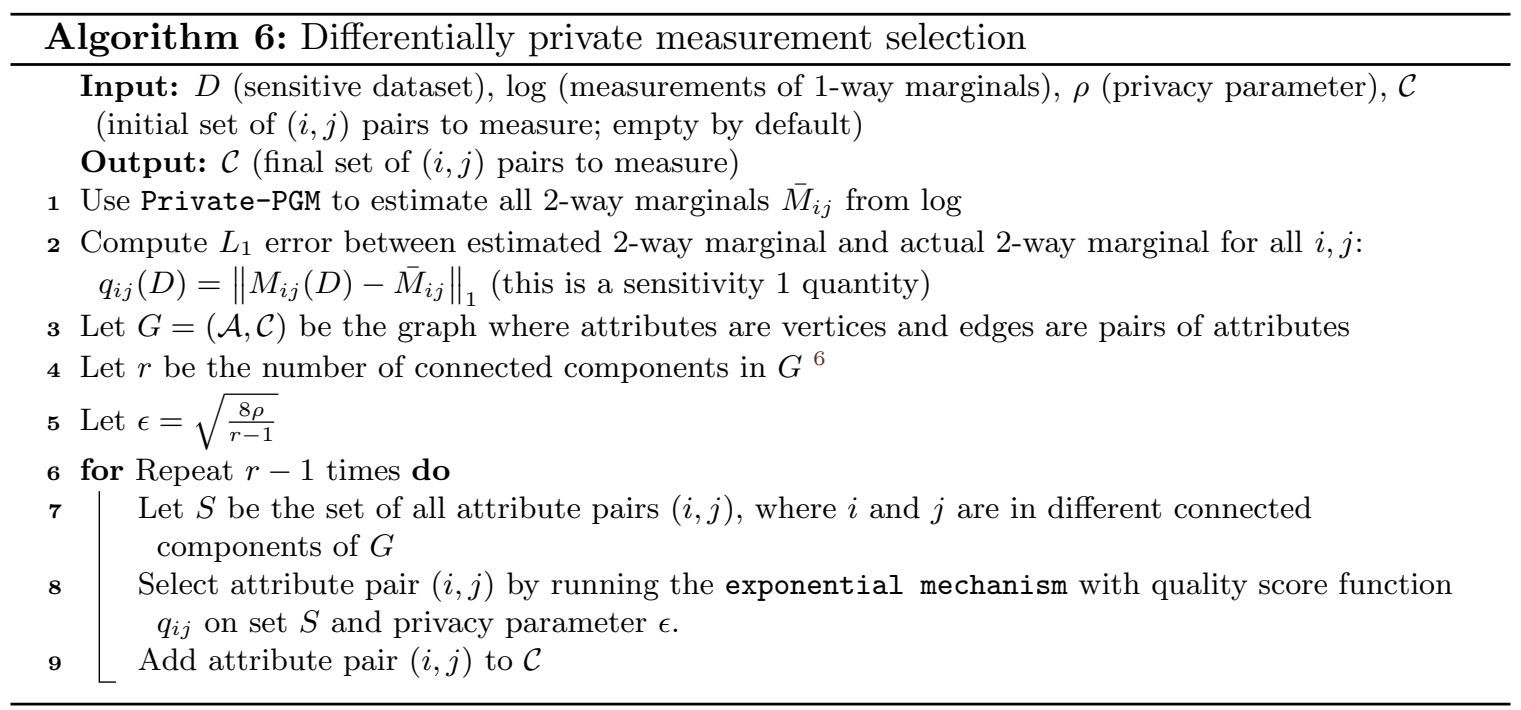

\section{Extensions}

One limitation with NIST-MST is that it is highly tailored to the setting of the NIST competition, and crucially relies on the existence of a public provisional dataset that can be used to select marginals. In more general settings, we will not always have access to a provisional dataset that follows a similar distribution as the sensitive data. For that reason, we propose MST, a general purpose mechanism that is inspired by the NIST-MST mechanism, but doesn't rely on the existence of provisional data. The basic mechanism is the same as NIST-MST outlined in Algorithm 2, with a couple minor exceptions. First, the preprocessing transformations and corresponding reverse transformations are not done those were specific to the U.S. Census dataset used in the competition and not generally applicable beyond that setting. Second, the measurement selection step, which previously relied on a provisional dataset to select correlated marginals, is replaced by a differentiallyprivate version that uses the sensitive dataset. MST devotes $\frac{1}{3}$ of the RDP budget towards measurement selection, and uses the remaining $\frac{2}{3}$ of the RDP budget for measuring the marginals. Privacy of MST follows by adaptive composition Proposition 3. For completeness, this calculation is given in the appendix.

The measurement selection algorithm is shown in Algorithm 6. Just like Algorithm 5, this algorithm tries to find a collection of attribute pairs that form a maximum spanning tree of an underlying correlation graph. However, as it uses the sensitive dataset, it must do this in a differentially private way. To achieve this, we first use a low-sensitivity approximation of the mutual information for assigning edge weights. We assume that we already measured all 1-way marginals, so we can get reasonable estimates of 2-way marginals by invoking Private-PGM. ${ }^{7}$ The edge weights are then computed as the $L_{1}$ distance between the true 2 -way marginal and the estimated one (a sensitivity 1 quantity). After computing the edge weights, Algorithm 6 can be seen as a differentially private version of Kruskal's algorithm [33] for computing a maximum spanning tree. It consists of $d-1$ steps (the number of edges in a

\footnotetext{
${ }^{6}$ If $\mathcal{C}$ is empty, this is just the number of attributes $d$.

${ }^{7}$ In this simple case, Private-PGM estimates 2-way marginals under an independence assumption, which could alternatively be achieved by multiplying the (noisy) one-way marginals together.
} 
spanning tree), and in each step, it adds a highly weighted edge that connects two different connected components. In Kruskal's algorithm, the highest weighted edge is chosen, but this would not be differentially private. We instead invoke the exponential mechanism to select a highly weighted edge in a differentially private way. In principle, we could apply any private selection algorithm here, including report-noisy-max [20] and the recently developed permute-and-flip mechanism [43]. While permute-and-flip is known to dominate the exponential mechanism under $\epsilon$-DP [43], the exponential mechanism enjoys a tighter privacy analysis under Rényi-DP [11].

The result of this algorithm is a collection of attribute pairs that will be measured by MST. Algorithm 6 has an optional argument, $\mathcal{C}$, which is an initial set of attribute pairs to measure. If this is supplied, the algorithm will always include those in the result, and then constructs a maximum spanning tree around them. This enables some extra flexibility that may be beneficial in certain settings where some marginals are more important than others, and need to be preserved even if they are not the most highly correlated. For many applications, $\mathcal{C}$ can just be empty. In the context of the competition, this feature is useful because the marginals relating SEX, CITY, and INCWAGE $\mathrm{A}_{\mathrm{A}}$ are very important (since their accuracy determines $\frac{1}{3}$ of the final score).

Theorem 3 (Privacy of Algorithm 6). Algorithm 6 is $(\alpha, \alpha \rho)$-RDP for all $\alpha \geq 1$.

Proof. Step $4 a$ is $\left(\alpha, \alpha \frac{1}{8} \epsilon^{2}\right)$-RDP by Proposition 2. Substituting $\epsilon=\sqrt{\frac{8 \rho}{r-1}}$, we see that it is equivalent to $\left(\alpha, \alpha \frac{\rho}{r-1}\right)$-RDP. It is called $r-1$ times, so the entire mechanism is $(\alpha, \alpha \rho)$-RDP by Proposition 3.

\section{EXPERIMENTS}

In this section we discuss the experimental evaluation carried out by the contest organizers, and how NIST-MST compared to the submissions from other teams. We offer our own insights into the numbers and explanations for the differences between mechanisms.

Evaluations were carried out on U.S. Census data for two different states: Arizona and Vermont. These datasets had 293,999 and 211,228 records, respectively. Scores were calculated separately for each of the three evaluation metrics described in Section 3.3. The final score was calculated by averaging the scores for each metric, each value of $\epsilon$, and each of the two datasets. In Table 5 we show the score breakdown by metric (averaged over Arizona and Vermont) for the top five submitted algorithms. We also evaluated our new mechanism (MST) and included it as an extra row (highlighted), even though it was not evaluated by the contest organizers at the time of the competition. This was possible because the final evaluation datasets were released after the competition, and the script used to evaluate the synthetic data was provided as part of the competitor pack.

MST was described in Section 6. We instantiate it with $\mathcal{C}=\left\{(\mathrm{SEX}, \mathrm{CITY}),\left(\mathrm{SEX}\right.\right.$, INCWAGE $\left._{\mathrm{A}}\right)$, $\left.\left(\mathrm{CITY}, \mathrm{INCWAGE}_{\mathrm{A}}\right)\right\}$, and add $\left(\mathrm{SEX}, \mathrm{CITY}, \mathrm{INCWAGE}_{\mathrm{A}}\right)$ to the returned result as well. These marginals are weighted using the same formula as NIST-MST (see Algorithm 5).

Among the contest submissions, NIST-MST consistently performed the best, for most metrics and values of $\epsilon$. Compared to DPSyn, it did a much better job at answering 3-way marginals and high order conjunctions, but performed slightly worse at handling the income inequality metric. Compared to every other mechanism, NIST-MST did better on every metric. 


\begin{tabular}{cc|ccc|c}
\hline$\epsilon$ & Team & $\begin{array}{c}3 \text {-way } \\
\text { marginals }\end{array}$ & $\begin{array}{c}\text { High order } \\
\text { conjunctions }\end{array}$ & $\begin{array}{c}\text { Income } \\
\text { inequality }\end{array}$ & Overall \\
\hline 0.3 & RMcKenna (NIST-MST) & 0.12 & 0.17 & 0.10 & 0.13 \\
0.3 & MST & $\mathbf{0 . 1 1}$ & $\mathbf{0 . 1 6}$ & 0.10 & $\mathbf{0 . 1 2}$ \\
0.3 & DPSyn & 0.15 & 0.31 & $\mathbf{0 . 0 7}$ & 0.18 \\
0.3 & PrivBayes & 0.19 & 0.29 & 0.18 & 0.22 \\
0.3 & Gardn999 & 0.21 & 0.32 & 0.25 & 0.26 \\
0.3 & UCLANESL & 0.57 & 0.72 & 0.22 & 0.50 \\
\hline 1.0 & RMcKenna (NIST-MST) & $\mathbf{0 . 0 9}$ & $\mathbf{0 . 1 5}$ & $\mathbf{0 . 0 4}$ & $\mathbf{0 . 0 9}$ \\
1.0 & MST & $\mathbf{0 . 0 9}$ & $\mathbf{0 . 1 5}$ & 0.05 & 0.10 \\
1.0 & DPSyn & 0.11 & 0.23 & 0.05 & 0.13 \\
1.0 & PrivBayes & 0.17 & 0.26 & 0.09 & 0.17 \\
1.0 & Gardn999 & 0.18 & 0.28 & 0.22 & 0.23 \\
1.0 & UCLANESL & 0.42 & 0.53 & 0.28 & 0.41 \\
\hline 8.0 & RMcKenna (NIST-MST) & $\mathbf{0 . 0 7}$ & $\mathbf{0 . 1 4}$ & 0.04 & $\mathbf{0 . 0 8}$ \\
8.0 & MST & 0.08 & $\mathbf{0 . 1 4}$ & 0.05 & 0.09 \\
8.0 & DPSyn & 0.09 & 0.20 & $\mathbf{0 . 0 2}$ & 0.10 \\
8.0 & PrivBayes & 0.13 & 0.23 & 0.09 & 0.15 \\
8.0 & Gardn999 & 0.17 & 0.26 & 0.24 & 0.22 \\
8.0 & UCLANESL & 0.35 & 0.41 & 0.25 & 0.34 \\
\hline
\end{tabular}

Table 5. Evaluation of NIST-MST and other mechanisms from competing teams, broken down by the three scoring metrics: 3-way marginals, high-order conjunctions, and income inequality. MST is also shown for comparison, even though that mechanism was not submitted during the competition. If it was submitted instead of NIST-MST, it would have placed first overall.

Generally speaking, NIST-MST and DPSyn (and to a lesser extent PrivBayes) seemed to be the only mechanisms that scored well on the income inequality metric, which is surprising given that it was the simplest metric and only required preserving one 3 -way marginal accurately. This raises an important point: mechanisms that understood the evaluation criteria well, and designed their mechanisms around it, generally performed better than mechanisms that just tried to generate good synthetic data without thinking about how its utility would be evaluated. NIST-MST and DPSyn did a good job of designing their mechanism for the task at hand, which was an important contributing factor for why they outperformed the other solutions.

While NIST-MST relied heavily on the provisional dataset for measurement selection, the more general variant MST still performs well, without explicitly relying on the provisional data. In fact, MST would have won first place if it was submitted instead of NIST-MST at the time of the competition; it was only slightly worse than NIST-MST and still better than the other submissions. The difference in performance between MST and NIST-MST was at most 0.01 for every metric and privacy budget evaluated.

At $\epsilon=0.3$, MST even achieved a smaller overall score than NIST-MST. NIST-MST measures both 2- and 3-way marginals, while MST only measures 2-way marginals. Since the privacy budget is relatively small, it makes sense that fewer measurements would work better here. 
A promising direction for future work is to adaptively select the number of marginals to measure based on the privacy budget and the amount of data available.

For a much more comprehensive evaluation of these mechanisms, we refer the reader to [9], which goes well beyond the metrics used in the competition to evaluate these mechanisms. This work is also a useful resource that summarizes each mechanism at a high level. Their results generally show that NIST-MST was the best performing mechanism for many of the additional evaluation metrics not used as part of the official scoring criteria. This suggests that NIST-MST produces the most generally useful synthetic data among the submitted mechanisms.

\section{Related Work}

Differentially private synthetic data has been an active area of research for several years. One of the earliest mechanisms proposed for this task was the "small database mechanism" [20], which instantiates the exponential mechanism over a set of small databases to select one that is statistically similar to the true data. Unfortunately, this mechanism is not able to run in practical settings, as it requires enumerating all possible datasets of a fixed sizes, resulting in a combinatorial explosion even for small dataset sizes (especially if the domain size is large).

In the competition, the top four submissions all followed the same basic template outlined in Figure 1: they selected and measured a collection of marginals, and then used those to estimate synthetic data. Moreover this approach has been applied more generally in the literature $[8,13,67,72]$. One key difference that sets our approach apart is Private-PGM. While $[13,67]$ do leverage graphical models to generate synthetic data, their approach is limited in how it makes use of the noisy measurements: they more or less treat the noisy marginals as the true marginals (with some lightweight post-processing), whereas Private-PGM makes use of all available measurements to resolve inconsistencies and boost utility in a principled manner. An alternative method for resolving inconsistencies and generating synthetic data from noisy marginals is proposed in [72]. This method does not construct an intermediate representation of the data distribution as Private-PGM does. As a result, their consistency resolution step only ensures local consistency (i.e., that all marginals internally agree on the common marginals), and does not satisfy the stronger notion of global consistency (i.e., that there is a data distribution that has all stated marginals), as Private-PGM does.

Another popular class of approaches for differentially private synthetic data is based on generative adversarial networks (GANs) [26]. Several differentially private GANs have been proposed for the purpose of generating synthetic data $[2,7,22,31,53-55,60,71]$. In fact, two teams in the NIST competition adopted a GAN-based approach (UCLANESL in Table 5, and one other team that did not place in the top five), however, their scores were not generally competitive with the other approaches that used the marginal-based framework. GANs are notoriously hard to train in practice [50] and when differential privacy constraints are enforced, it is even more difficult. It typically requires running an algorithm like DP-SGD [1] to train, and if it fails to converge (which is common) the privacy budget used for training is essentially wasted. Setting the right hyper-parameters is also a major challenge for this approach.

Another important and related problem is how to evaluate the quality of synthetic data $[4,9,29,51]$. Beyond the metrics used in the NIST competition, one alternative is 
pMSE, which is a general measure of distributional similarity [51]. Another alternative measure is machine learning efficacy, or how well the synthetic data supports machine learning applications [29]. A number of other measures for evaluating synthetic data can be found in [9]. We believe that there may be no universal answer to this question: it should ultimately depend on the data and its use cases. In general, it would be nice to have a mechanism that can automatically adapt to an analyst-provided workload, and generate synthetic data that provides high utility on the queries and tasks in that workload. Several workload-adaptive mechanisms exist, but they are generally restricted to settings where the full high-dimensional histogram can be explicitly materialized in vector form, and are thus unable to scale to high-dimensional domains $[20,23,27,35,40]$. When combined with Private-PGM, the scalability (and utility) of some of these mechanisms can be improved, however [42].

\section{Conclusions}

In this paper, we described NIST-MST, the winning mechanism from the NIST differential privacy synthetic data competition, and MST a new mechanism inspired by NIST-MST that works almost as well, without relying on public provisional data. While these mechanisms are state-of-the-art, the problem of differentially private synthetic data is far from solved. Nevertheless, we believe our basic framework centered around Private-PGM can serve as a core component of new mechanisms for this task. Private-PGM allows the mechanism designer to focus on what to measure, rather than how to post-process those measurements to get synthetic data while extracting the most utility from them. In fact, in the final round of the recently completed follow-up challenge, the NIST 2020 Temporal Map Challenge, both the first and second place teams used Private-PGM for post-processing, with each team developing novel techniques for measurement selection. 


\section{REFERENCES}

[1] M. Abadi, A. Chu, I. Goodfellow, H. B. McMahan, I. Mironov, K. Talwar, and L. Zhang. Deep learning with differential privacy. In Proceedings of the 2016 ACM SIGSAC conference on computer and communications security, 2016. https://doi.org/10.1145/2976749.2978318.

[2] N. C. Abay, Y. Zhou, M. Kantarcioglu, B. M. Thuraisingham, and L. Sweeney. Privacy preserving synthetic data release using deep learning. In M. Berlingerio, F. Bonchi, T. Gärtner, N. Hurley, and G. Ifrim, editors, Machine Learning and Knowledge Discovery in Databases - European Conference, ECML PKDD 2018, Dublin, Ireland, September 10-14, 2018, Proceedings, Part I, volume 11051 of Lecture Notes in Computer Science. Springer, 2018, 11051:510-526. https://doi.org/10.1007/978-3-030-10925-7\ -31.

[3] G. Ács, C. Castelluccia, and R. Chen. Differentially private histogram publishing through lossy compression. In ICDM, 2012.

[4] C. Arnold and M. Neunhoeffer. Really useful synthetic data - A framework to evaluate the quality of differentially private synthetic data. CoRR, abs/2004.07740, 2020. https://arxiv.org/abs/2004.07740, arXiv: 2004.07740.

[5] H. J. Asghar, M. Ding, T. Rakotoarivelo, S. Mrabet, and M. A. Kâafar. Differentially private release of high-dimensional datasets using the gaussian copula. CoRR, abs/1902.01499, 2019. http://arxiv. org/ abs/1902.01499, arXiv:1902.01499.

[6] B. Balle and Y.-X. Wang. Improving the gaussian mechanism for differential privacy: Analytical calibration and optimal denoising. In International Conference on Machine Learning, 2018. http: //proceedings.mlr.press/v80/balle18a.html.

[7] B. K. Beaulieu-Jones, Z. S. Wu, C. Williams, R. Lee, S. P. Bhavnani, J. B. Byrd, and C. S. Greene. Privacypreserving generative deep neural networks support clinical data sharing. Circulation: Cardiovascular Quality and Outcomes, 12(7):e005122, 2019. https://doi.org/10.1161/CIRCOUTCOMES.118.005122.

[8] V. Bindschaedler, R. Shokri, and C. A. Gunter. Plausible deniability for privacy-preserving data synthesis. Proceedings of the VLDB Endowment, 10(5):481-492, 2017. http://www.vldb.org/pvldb/ vol10/p481-bindschaedler.pdf, https://doi.org/10.14778/3055540.3055542.

[9] C. M. Bowen and J. Snoke. Comparative study of differentially private synthetic data algorithms from the NIST PSCR differential privacy synthetic data challenge. Journal of Privacy and Confidentiality, 11(1), Feb. 2021. https://doi.org/10.29012/jpc.748.

[10] M. Bun and T. Steinke. Concentrated differential privacy: Simplifications, extensions, and lower bounds. In Theory of Cryptography Conference. Springer, 2016. https ://doi .org/10.1007/978-3-662-53641-4_ 24.

[11] M. Cesar and R. Rogers. Bounding, concentrating, and truncating: Unifying privacy loss composition for data analytics. In V. Feldman, K. Ligett, and S. Sabato, editors, Proceedings of the 32nd International Conference on Algorithmic Learning Theory, volume 132 of Proceedings of Machine Learning Research. PMLR, 16-19 Mar 2021, 132:421-457. https://proceedings.mlr.press/v132/cesar21a.html.

[12] A. Charest. How can we analyze differentially-private synthetic datasets? Journal of Privacy and Confidentiality, 2(2), 2011. https://doi.org/10.29012/jpc.v2i2.589.

[13] R. Chen, Q. Xiao, Y. Zhang, and J. Xu. Differentially private high-dimensional data publication via sampling-based inference. In Proceedings of the 21th ACM SIGKDD International Conference on Knowledge Discovery and Data Mining. ACM, 2015. https://doi.org/10.1145/2783258.2783379.

[14] C. K. Chow and C. N. Liu. Approximating discrete probability distributions with dependence trees. IEEE transactions on Information Theory, 14(3):462-467, 1968. https://doi.org/10.1109/TIT.1968. 1054142 .

[15] G. Cormode, C. M. Procopiuc, D. Srivastava, E. Shen, and T. Yu. Differentially private spatial decompositions. In A. Kementsietsidis and M. A. V. Salles, editors, IEEE 28th International Conference on Data Engineering (ICDE 2012), Washington, DC, USA (Arlington, Virginia), 1-5 April, 2012. IEEE Computer Society, 2012. https://doi.org/10.1109/ICDE.2012.16.

[16] B. Ding, M. Winslett, J. Han, and Z. Li. Differentially private data cubes: optimizing noise sources and consistency. In T. K. Sellis, R. J. Miller, A. Kementsietsidis, and Y. Velegrakis, editors, Proceedings of the ACM SIGMOD International Conference on Management of Data, SIGMOD 2011, Athens, Greece, June 12-16, 2011. ACM, 2011. https://doi.org/10.1145/1989323.1989347.

[17] I. Dinur and K. Nissim. Revealing information while preserving privacy. In F. Neven, C. Beeri, and T. Milo, editors, Proceedings of the Twenty-Second ACM SIGACT-SIGMOD-SIGART Symposium on 
Principles of Database Systems, June 9-12, 2003, San Diego, CA, USA. ACM, 2003. https://doi.org/ 10.1145/773153.773173.

[18] J. Dong, A. Roth, and W. Su. Gaussian differential privacy. Journal of the Royal Statistical Society, 2021. http://arxiv.org/abs/1905.02383.

[19] C. Dwork, F. M. K. Nissim, and A. Smith. Calibrating noise to sensitivity in private data analysis. In TCC, 2006. https://doi.org/10.29012/jpc.v7i3.405.

[20] C. Dwork and A. Roth. The Algorithmic Foundations of Differential Privacy. Found. and Trends in Theoretical Computer Science, 2014. https://doi.org/10.1561/0400000042.

[21] V. Feldman, I. Mironov, K. Talwar, and A. Thakurta. Privacy amplification by iteration. In M. Thorup, editor, 59th IEEE Annual Symposium on Foundations of Computer Science, FOCS 2018, Paris, France, October 7-9, 2018. IEEE Computer Society, 2018. https://doi.org/10.1109/FOCS.2018.00056.

[22] L. Frigerio, A. S. de Oliveira, L. Gomez, and P. Duverger. Differentially private generative adversarial networks for time series, continuous, and discrete open data. In G. Dhillon, F. Karlsson, K. Hedström, and A. Zúquete, editors, ICT Systems Security and Privacy Protection - 34th IFIP TC 11 International Conference, SEC 2019, Lisbon, Portugal, June 25-27, 2019, Proceedings, volume 562 of IFIP Advances in Information and Communication Technology. Springer, 2019, 562:151-164. https://doi.org/10.1007/ 978-3-030-22312-0\_11.

[23] M. Gaboardi, E. J. G. Arias, J. Hsu, A. Roth, and Z. S. Wu. Dual query: Practical private query release for high dimensional data. In Proceedings of the 31th International Conference on Machine Learning, ICML 2014, Beijing, China, 21-26 June 2014, volume 32 of JMLR Workshop and Conference Proceedings. JMLR.org, 2014, 32:1170-1178. http://proceedings.mlr.press/v32/gaboardi14.html.

[24] S. L. Garfinkel, J. M. Abowd, and C. Martindale. Understanding database reconstruction attacks on public data. Communications of the ACM, 62(3):46-53, 2019. https://doi.org/10.1145/3287287.

[25] C. Ge, S. Mohapatra, X. He, and I. F. Ilyas. Kamino: Constraint-aware differentially private data synthesis. Proceedings of the VLDB Endowment, 14(10):1886-1899, 2021. http://www.vldb.org/pvldb/ vol14/p1886-ge.pdf.

[26] I. J. Goodfellow, J. Pouget-Abadie, M. Mirza, B. Xu, D. Warde-Farley, S. Ozair, A. C. Courville, and Y. Bengio. Generative adversarial nets. In Z. Ghahramani, M. Welling, C. Cortes, N. D. Lawrence, and K. Q. Weinberger, editors, Advances in Neural Information Processing Systems 27: Annual Conference on Neural Information Processing Systems 2014, December 813 2014, Montreal, Quebec, Canada, 2014. https://proceedings.neurips.cc/paper/2014/hash/ 5ca3e9b122f61f8f06494c97b1afccf3-Abstract.html.

[27] M. Hardt, K. Ligett, and F. McSherry. A simple and practical algorithm for differentially private data release. In P. L. Bartlett, F. C. N. Pereira, C. J. C. Burges, L. Bottou, and K. Q. Weinberger, editors, Advances in Neural Information Processing Systems 25: 26th Annual Conference on Neural Information Processing Systems 2012. Proceedings of a meeting held December 3-6, 2012, Lake Tahoe, Nevada, United States, 2012. https://proceedings.neurips.cc/paper/2012/hash/ 208e43f0e45c4c78cafadb83d2888cb6-Abstract.html.

[28] M. Hay, V. Rastogi, G. Miklau, and D. Suciu. Boosting the accuracy of differentially private histograms through consistency. PVLDB, 3(1-2):1021-1032, 2010. https://doi.org/10.14778/1920841.1920970.

[29] M. Hittmeir, A. Ekelhart, and R. Mayer. On the utility of synthetic data: An empirical evaluation on machine learning tasks. In Proceedings of the 14th International Conference on Availability, Reliability and Security, ARES 2019, Canterbury, UK, August 26-29, 2019. ACM, 2019. https://doi.org/10. 1145/3339252.3339281.

[30] Z. Huang, R. McKenna, G. Bissias, G. Miklau, M. Hay, and A. Machanavajjhala. Psyndb: accurate and accessible private data generation. Proceedings of the VLDB Endowment (Demo), 12(12):1918-1921, 2019. https://people.cs.umass.edu/ miklau/assets/pubs/dp/huang19psyndata-demo.pdf.

[31] J. Jordon, J. Yoon, and M. van der Schaar. PATE-GAN: generating synthetic data with differential privacy guarantees. In 7th International Conference on Learning Representations, ICLR 2019, New Orleans, LA, USA, May 6-9, 2019. OpenReview.net, 2019. https://openreview.net/forum?id=S1zk9iRqF7.

[32] D. Koller and N. Friedman. Probabilistic graphical models: principles and techniques. MIT press, 2009. https://doi.org/10.1017/S0269888910000275.

[33] J. B. Kruskal. On the shortest spanning subtree of a graph and the traveling salesman problem. Proceedings of the American Mathematical society, 7(1):48-50, 1956. https://doi.org/10.2307/2033241. 
[34] C. Li, M. Hay, G. Miklau, and Y. Wang. A data-and workload-aware algorithm for range queries under differential privacy. PVLDB, 7(5):341-352, 2014.

[35] C. Li, M. Hay, V. Rastogi, G. Miklau, and A. McGregor. Optimizing linear counting queries under differential privacy. In Proceedings of the twenty-ninth ACM SIGMOD-SIGACT-SIGART symposium on Principles of database systems. ACM, 2010. https://doi.org/10.1145/1807085.1807104.

[36] C. Li and G. Miklau. An adaptive mechanism for accurate query answering under differential privacy. $P V L D B, 5(6): 514-525,2012$.

[37] C. Li, G. Miklau, M. Hay, A. McGregor, and V. Rastogi. The matrix mechanism: optimizing linear counting queries under differential privacy. The VLDB Journal, 24(6):757-781, 2015. https://doi.org/ $10.1007 / \mathrm{s} 00778-015-0398-\mathrm{x}$.

[38] H. Li, L. Xiong, and X. Jiang. Differentially private synthesization of multi-dimensional data using copula functions. In S. Amer-Yahia, V. Christophides, A. Kementsietsidis, M. N. Garofalakis, S. Idreos, and V. Leroy, editors, Proceedings of the 17th International Conference on Extending Database Technology, EDBT 2014, Athens, Greece, March 24-28, 2014. OpenProceedings.org, 2014. https://doi.org/10. 5441/002/edbt.2014.43.

[39] F. Liu. Model-based differentially private data synthesis. arXiv preprint arXiv:1606.08052, 2016. https: //arxiv.org/abs/1606.08052.

[40] R. McKenna, G. Miklau, M. Hay, and A. Machanavajjhala. Optimizing error of high-dimensional statistical queries under differential privacy. Proceedings of the VLDB Endowment, 11(10):1206-1219, 2018. https://doi.org/10.14778/3231751.3231769.

[41] R. McKenna, G. Miklau, and D. Sheldon. Private-pgm. Zenodo, (v2021-10-04-jpc), Oct. 2021. https: //doi.org/10.5281/zenodo.5548533.

[42] R. McKenna, D. Sheldon, and G. Miklau. Graphical-model based estimation and inference for differential privacy. In International Conference on Machine Learning, 2019. http://proceedings.mlr.press/v97/ mckenna19a.html.

[43] R. McKenna and D. R. Sheldon. Permute-and-flip: A new mechanism for differentially private selection. In H. Larochelle, M. Ranzato, R. Hadsell, M. Balcan, and H. Lin, editors, Advances in Neural Information Processing Systems 33: Annual Conference on Neural Information Processing Systems 2020, NeurIPS 2020, December 6-12, 2020, virtual, 2020. https://proceedings.neurips.cc/paper/2020/ hash/01e00f 2 f 4 bf cbb7505cb641066f 2859b-Abstract.html.

[44] F. McSherry and K. Talwar. Mechanism design via differential privacy. In FOCS, 2007. https://doi. org/10.1145/2090236.2090254.

[45] I. Mironov. Rényi differential privacy. In 2017 IEEE 30th Computer Security Foundations Symposium (CSF). IEEE, 2017. https://doi.org/10.1109/CSF.2017.11.

[46] W. Qardaji, W. Yang, and N. Li. Differentially private grids for geospatial data. In Intl. Conference on Data Engineering (ICDE). IEEE, 2013.

[47] W. Qardaji, W. Yang, and N. Li. Understanding hierarchical methods for differentially private histograms. PVLDB, 6(14):1954-1965, 2013.

[48] W. H. Qardaji, W. Yang, and N. Li. Priview: practical differentially private release of marginal contingency tables. In C. E. Dyreson, F. Li, and M. T. Özsu, editors, International Conference on Management of Data, SIGMOD 2014, Snowbird, UT, USA, June 22-27, 2014. ACM, 2014. https: //doi.org/10.1145/2588555.2588575.

[49] S. Ruggles, S. Flood, S. Foster, R. Goeken, J. Pacas, M. Schouweiler, and M. Sobek. Ipums usa: Version 11.0 [dataset]. Technical report, IPUMS, 2021. https://doi.org/10.18128/D010.V11.0.

[50] T. Salimans, I. J. Goodfellow, W. Zaremba, V. Cheung, A. Radford, and X. Chen. Improved techniques for training gans. In D. D. Lee, M. Sugiyama, U. von Luxburg, I. Guyon, and R. Garnett, editors, Advances in Neural Information Processing Systems 29: Annual Conference on Neural Information Processing Systems 2016, December 5-10, 2016, Barcelona, Spain, 2016. https://proceedings.neurips . cc/paper/2016/hash/8a3363abe792db2d8761d6403605aeb7-Abstract.html.

[51] J. Snoke, G. M. Raab, B. Nowok, C. Dibben, A. Slavkovic, et al. General and specific utility measures for synthetic data. Journal of the Royal Statistical Society Series A, 181(3):663-688, 2018. https: //doi.org/10.1111/rssa.12358.

[52] D. M. Sommer, S. Meiser, and E. Mohammadi. Privacy loss classes: The central limit theorem in differential privacy. Proceedings on privacy enhancing technologies, 2019(2):245-269, 2019. https://doi. org/10.2478/popets-2019-0029. 
[53] U. Tantipongpipat, C. Waites, D. Boob, A. A. Siva, and R. Cummings. Differentially private mixed-type data generation for unsupervised learning. CoRR, abs/1912.03250, 2019. http://arxiv .org/abs/1912. 03250, arXiv: 1912.03250.

[54] A. Torfi, E. A. Fox, and C. K. Reddy. Differentially private synthetic medical data generation using convolutional gans. CoRR, abs/2012.11774, 2020. https://arxiv.org/abs/2012.11774, arXiv: 2012. 11774.

[55] R. Torkzadehmahani, P. Kairouz, and B. Paten. DP-CGAN: differentially private synthetic data and label generation. In IEEE Conference on Computer Vision and Pattern Recognition Workshops, CVPR Workshops 2019, Long Beach, CA, USA, June 16-20, 2019. Computer Vision Foundation / IEEE, 2019. http://openaccess.thecvf.com/content_CVPRW_2019/html/CV-COPS/Torkzadehmahani_DP-CGAN_ Differentially_Private_Synthetic_Data_and_Label_Generation_CVPRW_2019_paper.html, https://doi.org/10.1109/CVPRW.2019.00018.

[56] G. Vietri, G. Tian, M. Bun, T. Steinke, and Z. S. Wu. New oracle-efficient algorithms for private synthetic data release. In Proceedings of the 37th International Conference on Machine Learning, ICML 2020, 13-18 July 2020, Virtual Event, volume 119 of Proceedings of Machine Learning Research. PMLR, 2020, 119:9765-9774. http://proceedings.mlr.press/v119/vietri20b.html.

[57] www.nist.gov. 2018 differential privacy synthetic data challenge. https://www . nist.gov/ctl/pscr/open-innovation-prize-challenges/past-prize-challenges/ 2018-differential-privacy-synthetic, 2018.

[58] X. Xiao, G. Wang, and J. Gehrke. Differential privacy via wavelet transforms. IEEE Transactions on Knowledge and Data Engineering, 23(8):1200-1214, 2011.

[59] Y. Xiao, L. Xiong, L. Fan, S. Goryczka, and H. Li. DPCube: Differentially private histogram release through multidimensional partitioning. Transactions of Data Privacy, 7(3), 2014.

[60] L. Xie, K. Lin, S. Wang, F. Wang, and J. Zhou. Differentially private generative adversarial network. CoRR, abs/1802.06739, 2018. http://arxiv.org/abs/1802.06739, arXiv:1802.06739.

[61] C. Xu, J. Ren, Y. Zhang, Z. Qin, and K. Ren. Dppro: Differentially private high-dimensional data release via random projection. IEEE Transactions on Information Forensics and Security, 12(12):3081-3093, 2017. https://doi.org/10.1109/TIFS.2017.2737966.

[62] J. Xu, Z. Zhang, X. Xiao, Y. Yang, and G. Yu. Differentially private histogram publication. In Data Engineering (ICDE), 2012 IEEE 28th International Conference on, 2012. https://doi.org/10.1109/ ICDE. 2012.48.

[63] J. Xu, Z. Zhang, X. Xiao, Y. Yang, G. Yu, and M. Winslett. Differentially private histogram publication. The VLDB Journal, 2013. http://dx.doi.org/10.1007/s00778-013-0309-y, https://doi.org/ 10.1007/s00778-013-0309-y.

[64] G. Yaroslavtsev, G. Cormode, C. M. Procopiuc, and D. Srivastava. Accurate and efficient private release of datacubes and contingency tables. In ICDE, 2013.

[65] G. Yuan, Y. Yang, Z. Zhang, and Z. Hao. Convex optimization for linear query processing under approximate differential privacy. In Proceedings of the 22nd ACM SIGKDD International Conference on Knowledge Discovery and Data Mining. ACM, 2016.

[66] G. Yuan, Z. Zhang, M. Winslett, X. Xiao, Y. Yang, and Z. Hao. Low-rank mechanism: optimizing batch queries under differential privacy. PVLDB, 5(11):1352-1363, 2012.

[67] J. Zhang, G. Cormode, C. M. Procopiuc, D. Srivastava, and X. Xiao. Privbayes: Private data release via bayesian networks. ACM Transactions on Database Systems (TODS), 42(4):25:1-25:41, 2017. https: //doi.org/10.1145/3134428.

[68] J. Zhang, X. Xiao, and X. Xie. Privtree: A differentially private algorithm for hierarchical decompositions. In $S I G M O D, 2016$.

[69] W. Zhang, J. Zhao, F. Wei, and Y. Chen. Differentially private high-dimensional data publication via markov network. EAI Endorsed Trans. Security Safety, 6(19):e4, 2019. https://doi.org/10.4108/eai. 29-7-2019.159626.

[70] X. Zhang, R. Chen, J. Xu, X. Meng, and Y. Xie. Towards accurate histogram publication under differential privacy. In SDM, 2014. http://epubs.siam.org/doi/abs/10.1137/1.9781611973440.68, arXiv:http://epubs.siam.org/doi/pdf/10.1137/1.9781611973440.68, https://doi.org/10.1137/ 1.9781611973440 .68$.

[71] X. Zhang, S. Ji, and T. Wang. Differentially private releasing via deep generative model (technical report). arXiv preprint arXiv:1801.01594, 2018. https://arxiv .org/abs/1801.01594. 
[72] Z. Zhang, T. Wang, N. Li, J. Honorio, M. Backes, S. He, J. Chen, and Y. Zhang. Privsyn: Differentially private data synthesis. In 30th USENIX Security Symposium (USENIX Security 21). USENIX Association, Aug. 2021. https://www. usenix.org/conference/usenixsecurity21/presentation/zhang-zhikun.

[73] J. Zhao, T. Wang, T. Bai, K. Lam, X. Ren, X. Yang, S. Shi, Y. Liu, and H. Yu. Reviewing and improving the gaussian mechanism for differential privacy. CoRR, abs/1911.12060, 2019. http://arxiv.org/abs/ 1911.12060, arXiv: 1911.12060. 


\section{Appendix A. Supplementary Material}

Noise Calibration for MST. We begin by calculating our total RDP budget from $(\epsilon, \delta)$ by invoking Proposition 4. In particular, we find the largest value of $\rho$ such that $(\alpha, \alpha \rho)$-RDP implies $(\epsilon, \delta)$-DP by Proposition 4 . We accomplish this numerically. We will now divide our RDP budget " $\rho$ " equally among the three steps (4) and (5) and (6) of MST. To achieve $\frac{\rho}{3}$-RDP in steps (4) and (6), it suffices to set $\sigma=\sqrt{\frac{3}{2 \rho}}$ by Proposition 1 . To achieve $\frac{\rho}{3}$-RDP in step (5). we simplv call Aløorithm 6 with privacv parameter $\frac{\rho}{n}$. The correctness of this

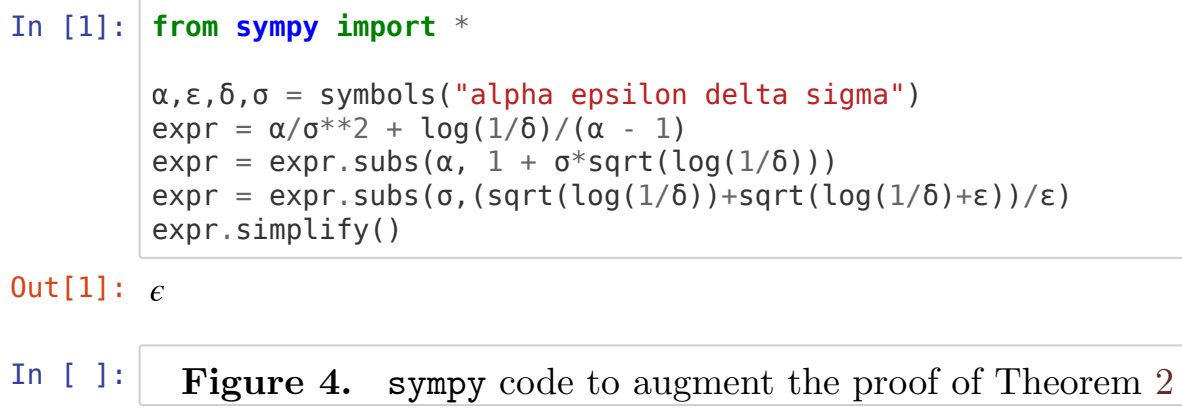

Additional Experiment on MST vs. NIST-MST. In Section 7, we evaluated MST in the context of the challenge test suite and evaluation metrics, and found that it performed comparably to NIST-MST. In this section, we compare the quality of the marginals selected from public data (Algorithm 5) and from the sensitive data (Algorithm 6) using various privacy budgets. Specifically, we logged the marginals selected by MST, and compared them to the publicly chosen marginals used in NIST-MST (ignoring the extra 2- and 3-way marginals selected in step 4) using the mutual information criteria described in Algorithm 5. We also show the scores that would be achieved by the best marginals (i.e., the true maximum spanning tree), as well as the scores that would be achieved by a random spanning tree. As shown in the table below, both the publicly chosen marginals and the privately chosen marginals nearly match the score acheived by the best marginals, for both the Arizona and Vermont datasets. The publicly chosen marginals are slightly better at $\epsilon=0.3$ and $\epsilon=1.0$, and slightly worse at $\epsilon=8.0$. Both variants are much better than the random baseline.

\begin{tabular}{c|cc} 
Selected Marginals & Arizona & Vermont \\
\hline Best & 73.64 & 63.32 \\
Public (Algorithm 5) & 72.81 & 62.79 \\
$\epsilon=0.3$ (Algorithm 6) & 70.53 & 61.15 \\
$\epsilon=1.0$ (Algorithm 6) & 72.27 & 62.35 \\
$\epsilon=8.0$ (Algorithm 6) & 73.05 & 63.09 \\
Random & 8.55 & 6.95
\end{tabular}



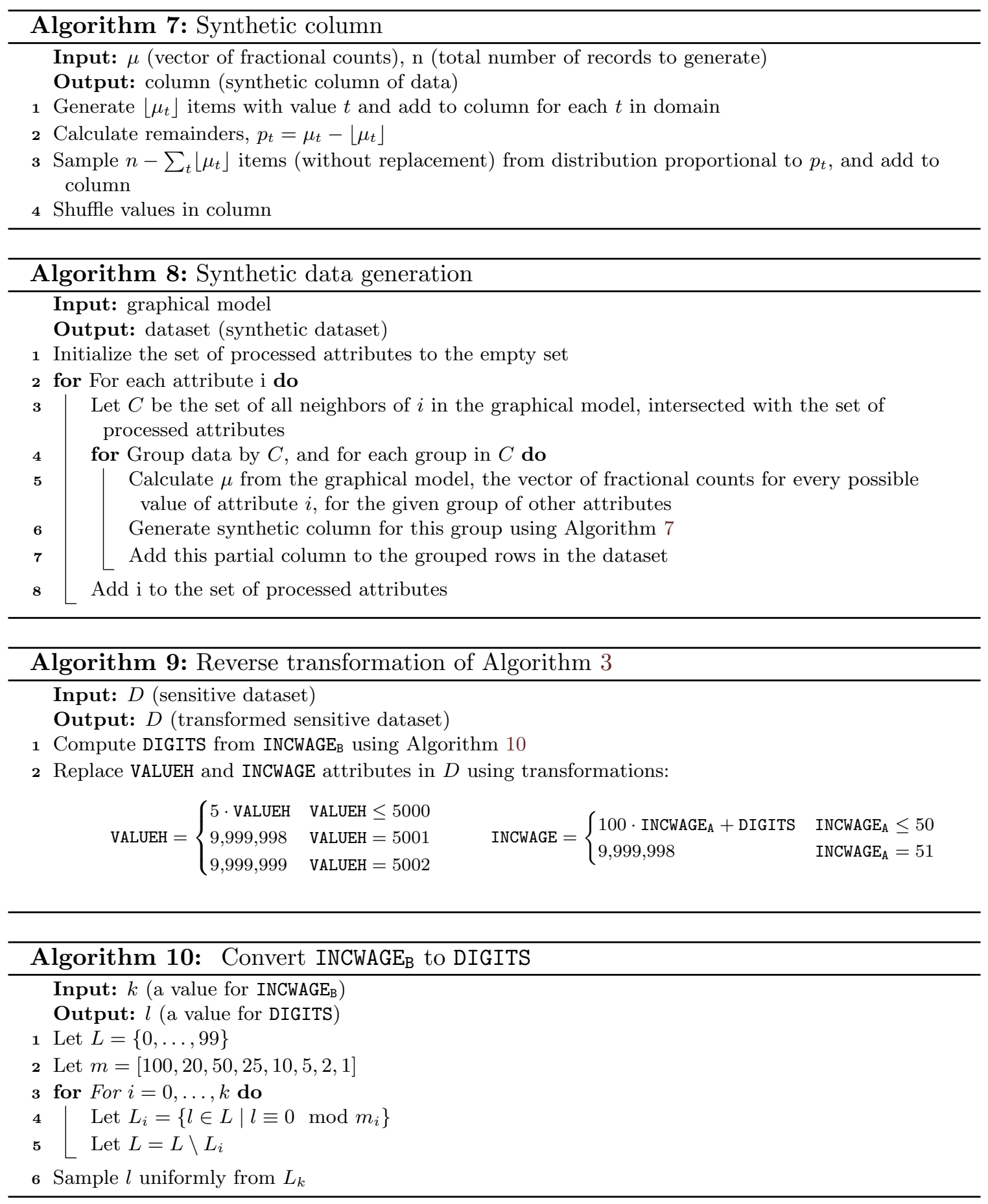
This work is licensed under the Creative Commons License Attribution-NonCommercial-NoDerivatives 4.0 International (CC BY-NC-ND 4.0). To view a copy of this license, visit https: //creativecommons. org/licenses/by-nc-nd/4.0/ or send a letter to Creative Commons, 171 Second St, Suite 300, San Francisco, CA 94105, USA, or Eisenacher Strasse 2, 10777 Berlin, Germany 